\title{
Global Optimization of Mixed-Integer Nonlinear Programs: A Theoretical and Computational Study*
}

\author{
Mohit Tawarmalani $^{\dagger} \quad$ Nikolaos V. Sahinidis ${ }^{\ddagger}$ \\ 30 December 1999. Revised: 19 October 2002, 31 March 2003.
}

\begin{abstract}
This work addresses the development of an efficient solution strategy for obtaining global optima of continuous, integer, and mixed-integer nonlinear programs. Towards this end, we develop novel relaxation schemes, range reduction tests, and branching strategies which we incorporate into the prototypical branch-and-bound algorithm.

In the theoretical/algorithmic part of the paper, we begin by developing novel strategies for constructing linear relaxations of mixed-integer nonlinear programs and prove that these relaxations enjoy quadratic convergence properties. We then use Lagrangian/linear programming duality to develop a unifying theory of domain reduction strategies as a consequence of which we derive many range reduction strategies currently used in nonlinear programming and integer linear programming. This theory leads to new range reduction schemes, including a learning heuristic that improves initial branching decisions by relaying data across siblings in a branch-and-bound tree. Finally, we incorporate these relaxation and reduction strategies in a branch-and-bound algorithm that incorporates branching strategies that guarantee finiteness for certain classes of continuous global optimization problems.

In the computational part of the paper, we describe our implementation discussing, wherever appropriate, the use of suitable data structures and associated algorithms. We present computational experience with benchmark separable concave quadratic programs, fractional $0-1$ programs, and mixed-integer nonlinear programs from applications in synthesis of chemical processes, engineering design, just-in-time manufacturing, and molecular design.
\end{abstract}

\footnotetext{
* The research was supported in part by ExxonMobil Upstream Research Company, National Science Foundation awards DMII 95-02722, BES 98-73586, ECS 00-98770, and CTS 01-24751, and the Computational Science and Engineering Program of the University of Illinois.

$\dagger$ Krannert School of Management, Purdue University, mtawarma@mgmt.purdue.edu.

${ }_{\ddagger}^{\ddagger}$ Department of Chemical and Biomolecular Engineering, University of Illinois at UrbanaChampaign.

$\S$ Corresponding author (email: nikos@uiuc.edu).
} 


\section{Introduction}

Research in optimization attracted attention when significant advances were made in linear programming in the late 1940's. Motivated by applications, developments in nonlinear programming followed quickly and concerned themselves mostly with local optimization guaranteeing globality only under certain convexity assumptions. However, problems in engineering design, logistics, manufacturing, and the chemical and biological sciences often demand modeling via nonconvex formulations that exhibit multiple local optima. The potential gains to be obtained through global optimization of these problems motivated a stream of recent efforts, including the development of deterministic and stochastic global optimization algorithms.

Solution strategies explicitly addressing mixed-integer nonlinear programs (MINLPs) have appeared rather sporadically in the literature and initially dealt mostly with problems that are convex when integrality restrictions are dropped and/or contain nonlinearities that are quadratic $[24,22,16,23,10,6,7,5]$. A few recent works have indicated that the application of deterministic branchand-bound algorithms to the global optimization of general classes of MINLPs is promising $[30,37,12,36]$. In this paper, we demonstrate that branch-and-bound is a viable tool for global optimization of purely continuous, purely integer, as well as mixed-integer nonlinear programs if supplemented with appropriate range reduction tools, relaxation schemes, and branching strategies.

The application of the prototypical branch-and-bound algorithm to continuous spaces is outlined in Section 2 and contrasted to application in discrete spaces. The remainder of the paper is devoted to developing theory and methodology for various steps of the branch-and-bound algorithm as applied to MINLP problems. In Section 3, we synthesize a novel linear programming lower bounding scheme by combining factorable programming techniques [26] with the sandwich algorithm $[13,29]$. In the context of the latter algorithm, we propose a new variant that is based on a projective error rule and prove that this variant exhibits quadratic convergence.

In Section 4, we develop a new framework for range reduction and show that many existing range reduction schemes in integer and nonlinear programming $[41,18,17,20,34,30,31,35,43]$ are special cases of our approach. Additionally, our reduction framework naturally leads to a novel learning reduction heuristic that eliminates search space in the vicinity of fathomed nodes, thereby improving older branching decisions and expediting convergence.

In Section 5, we present a new rectangular partitioning procedure for nonlinear programs. In Section 6, we describe how the branch-and-bound tree can be efficiently navigated and expanded. Finally, in Section 7, we describe computational experience with the Branch-And-Reduce Optimization Navigator (BARON), which is the implementation of the proposed branch-and-bound global optimization algorithm. Computations are presented for continuous, integer, and mixed-integer nonlinear programs demonstrating that a large class

of difficult nonconvex optimization problems can be solved in an entirely automated fashion with the proposed techniques. 


\section{Branch-and-Bound in Continuous Spaces}

Consider the following mixed-integer nonlinear program:

$$
\begin{array}{ll}
\min & f(x, y) \\
\text { s.t. } & g(x, y) \leq 0 \\
& x \in X \subseteq \mathbb{R}^{n} \\
& y \in Y \subseteq \mathbb{Z}^{p}
\end{array}
$$

where $f:(X, Y) \mapsto \mathbb{R}$ and $g:(X, Y) \mapsto \mathbb{R}^{m}$. We restrict attention to factorable functions $f$ and $g$, i.e., functions that are recursive sums and products of univariate functions. This class of functions suffices to describe most application areas of interest [26]. Examples of factorable functions include $f\left(x_{1}, x_{2}\right)=$ $x_{1} x_{2}, f\left(x_{1}, x_{2}\right)=x_{1} / x_{2}, f\left(x_{1}, x_{2}, x_{3}, x_{4}\right)=\sqrt{\exp \left(x_{1} x_{2}+x_{3} \ln x_{4}\right) x_{3}^{3}}, f\left(x_{1}, x_{2}\right.$, $\left.x_{3}, x_{4}\right)=\left(x_{1}^{2} x_{2}^{0.3} x_{3}\right) / x_{4}^{2}+\exp \left(x_{1}^{2} x_{4} / x_{2}\right)-x_{1} x_{2}, f(x)=\sum_{i=1}^{n} \ln _{i}\left(x_{i}\right)$, and $f(x)=\sum_{i=1}^{T} \prod_{j=1}^{p_{i}}\left(c_{i j}^{0}+c_{i j} x\right)$ with $x \in \mathbb{R}^{n}, c_{i j} \in \mathbb{R}^{n}$ and $c_{i j}^{0} \in \mathbb{R}(i=$ $\left.1, \ldots, T ; j=1, \ldots, p_{i}\right)$.

Initially conceived as an algorithm to solve combinatorial optimization problems $[21,9]$, branch-and-bound has evolved to a method for solving more general multi-extremal problems like $\mathrm{P}[11,19]$. To solve $\mathrm{P}$, branch-and-bound computes lower and upper bounds on the optimal objective function value over successively refined partitions of the search space. Partition elements are generated and placed on a list of open partition elements. Elements from this list are selected for further processing and further partitioning, and are deleted when their lower bounds are no lower than the best known upper bound for the problem.

A bounding scheme is called consistent if any unfathomed partition element can be further refined and, for any sequence of infinitely decreasing partition elements, the upper and lower bounds converge in the limit. Most algorithms ensure consistency through an exhaustive partitioning strategy, i.e., one that guarantees that partition elements converge to points or other sets over which $\mathrm{P}$ is easily solvable. A selection scheme is called bound-improving if a partition with the current lowest bound is chosen after a finite number of steps for further bounding and partitioning. If the bounding scheme is consistent and the selection scheme is bound-improving, branch-and-bound is convergent [19].

Remark 2.1. In 0-1 programming, branching on a binary variable creates two subproblems in both of which that variable is fixed. On the contrary, branching on a continuous variable in nonlinear programming may require infinitely many subdivisions. As a result, branch-and-bound for 0-1 programs is finite but merely convergent for continuous nonlinear programs. Results pertaining to finite rectangular branching schemes for continuous global optimization problems have been rather sparse in the literature [35, 3, 1]. Finiteness for these problems was obtained based on branching schemes that are exhaustive and branch on the incumbent, and/or explicitly took advantage of special problem structure. 
Remark 2.2. While dropping the integrality conditions leads to a linear relaxation of an MILP, dropping integrality conditions may not suffice to obtain a useful relaxation of an MINLP. Indeed, the resulting NLP may involve nonconvexities that make solution difficult. It is even possible that the resulting NLP may be harder to solve to global optimality than the original MINLP.

Remark 2.3. Linear programming technology has reached a level of maturity that provides robust and reliable software for solving linear relaxations of MILP problems. Nonlinear programming solvers, however, often fail even in solving convex problems. At the theoretical level, duality theory provides necessary and sufficient conditions for optimality in linear programming, whereas the KKT optimality conditions, that are typically exploited by nonlinear programming algorithms, are not even necessary unless certain constraint qualifications hold.

In the sequel, we address the development of branch-and-bound algorithms for MINLPs. Branch-and-bound is more of a prototype of a global optimization algorithm than a formal algorithmic specification since it employs a number of schemes that may be tailored to the application at hand. In order to derive an efficient algorithm, it is necessary to study the various techniques for relaxation construction, domain reduction, upper bounding, partitioning, and node selection. Also, a careful choice of data structures and associated algorithms is necessary for developing an efficient implementation. We develop theory and methodology behind these algorithmic components in the next four sections.

\section{Lower Bounding}

In this section, we address the question of constructing a lower bounding procedure for the factorable programming problem $\mathrm{P}$ stated in Section 2. The proposed methodology starts by using the following recursive algorithm to decompose factorable functions in terms of sums and products of univariate functions that are subsequently bounded to yield a relaxation of the original problem:

\section{Algorithm Relax $\mathbf{f}(\mathbf{x})$}

If $f(x)$ is a function of single variable $x \in\left[x^{l}, x^{u}\right]$, then

Construct under- and over-estimators for $f(x)$ over $\left[x^{l}, x^{u}\right]$,

else if $f(x)=g(x) / h(x)$, then

Fractional_Relax $(f, g, h)$

end of if

else if $f(x)=\prod_{i=1}^{t} f_{i}(x)$, then

for $i:=1$ to $t$ do

Introduce variable $y_{f_{i}}$, such that $y_{f_{i}}=\operatorname{Relax} f_{i}(x)$

end of for

Introduce variable $y_{f}$, such that $y_{f}=$ Multilinear_Relax $\prod_{i=1}^{t} y_{f_{i}}$ 
else if $f(x)=\sum_{i=1}^{t} f_{i}(x)$, then

for $i:=1$ to $t$ do

Introduce variable $y_{f_{i}}$, such that $y_{f_{i}}=\operatorname{Relax} f_{i}(x)$

end of for

Introduce variable $y_{f}$, such that $y_{f}=\sum_{i=1}^{t} y_{f_{i}}$

else if $f(x)=g(h(x))$, then

Introduce variable $y_{h}=$ Relax $h(x)$

Introduce variable $y_{f}=\operatorname{Relax} g\left(y_{h}\right)$

end of if

Note that while decomposing $f(x)$ as a product/sum of functions, a variable is introduced if and only if another variable corresponding to the same function has not been introduced at an earlier step.

Theorem 3.1 ([26]). Consider a function $f(x)=g(h(x))$ where $h: \mathbb{R}^{n} \mapsto \mathbb{R}$ and $g: \mathbb{R} \mapsto \mathbb{R}$. Let $S \subseteq \mathbb{R}^{n}$ be a convex domain of $h$ and let $a \leq h(x) \leq b$ over $S$. Assume $c(x)$ and $C(x)$ are, respectively, a convex underestimator and a concave overestimator of $h(x)$ over $S$. Also, let $e(\cdot)$ and $E(\cdot)$ be the convex and concave envelopes of $g(h)$ for $h \in[a, b]$. Let $h_{\min }$ and $h_{\max }$ be such that $g\left(h_{\min }\right)=\inf _{h \in[a, b]}(g(h))$ and $g\left(h_{\max }\right)=\sup _{h \in[a, b]}(g(h))$. Then,

$$
e\left(\operatorname{mid}\left[c(x), C(x), h_{\min }\right]\right)
$$

is a convex underestimating function and

$$
E\left(\operatorname{mid}\left[c(x), C(x), h_{\max }\right]\right)
$$

is a concave overestimating function for $f(x)$.

Theorem 3.2. The underestimator and overestimator in Theorem 3.1 are implied in the relaxation derived through the application of Algorithm Relax as long as $e(\cdot)$ and $E(\cdot)$ are used as the under-and over-estimators for $g\left(y_{h}\right)$.

Proof. Consider the $(n+2)$-dimensional space of points of the form $(x, h, f)$. Construct the surface $M=\{(x, h, f) \mid f=g(h)\}$, the set $F=\{(x, h, f) \mid$ $e(h) \leq f \leq E(h)\}$, and the set $H=\{(x, h, f) \mid c(x) \leq h \leq C(x), x \in S\}$. Then, the feasible region as constructed by Algorithm Relax is the projection of $F \cap H$ on the space of $x$ and $f$ variables assuming $h(x)$ does not occur elsewhere in the factorable program. The resulting set is convex since intersection and projection operations preserve convexity. For a given $x$, let us characterize the set of feasible points, $(x, f)$. Note that $(x, f) \in F \cap H$ as long as $f \geq f_{\min }=$ $\min \{e(h) \mid h \in[c(x), C(x)]\}$. If $h_{\min } \leq c(x)$, the minimum value of $e(h)$ is attained at $c(x)$, by quasiconvexity of $e$. Similarly, if $c(x) \leq h_{\min } \leq C(x)$, then the minimum is attained at $h_{\min }$ and, if $C(x) \leq h_{\min }$, then the minimum is attained at $C(x)$. The resulting function is exactly the same as described in Theorem 3.1. The overestimating function follows by a similar argument. 
We chose to relax the composition of functions in the way described in Algorithm Relax instead of constructing underestimators based on Theorem 3.1 because our procedure is simpler to implement, and may lead to tighter relaxations if $h(x)$ occurs more than once in $\mathrm{P}$. On the other hand, the scheme implied in Theorem 3.1 introduces one variable lesser than that in Algorithm Relax for every univariate function relaxed by the algorithm.

We now address the relaxation of special functions, beginning with the simple monomial $y=\prod_{i=1}^{t} y_{f_{i}}$. Various relaxation strategies are possible for simple monomials as well as more general multilinear functions. For example, in [40], a complete characterization of the generating set of the convex polyhedral encloser of a multilinear function over any hypercube is provided in terms of its extreme points. While this implies trivially the polyhedral description of the convex encloser of the product of $t$ variables (cf. Theorem 4.8, p. 96 [27]), this description is exponential in $t$. To keep the implementation simple, we instead employ a recursive arithmetic interval scheme for bounding the product.

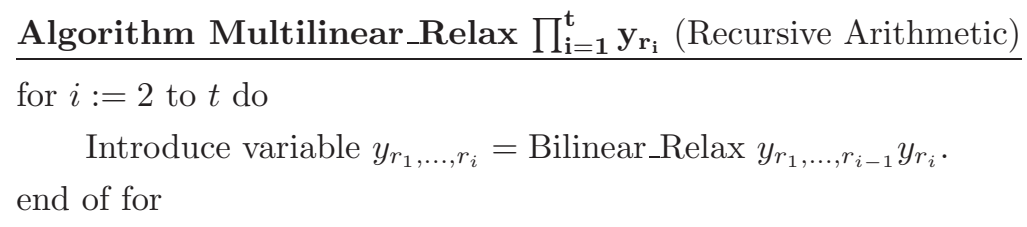

The fractional term is relaxed using the algorithm developed in [39].

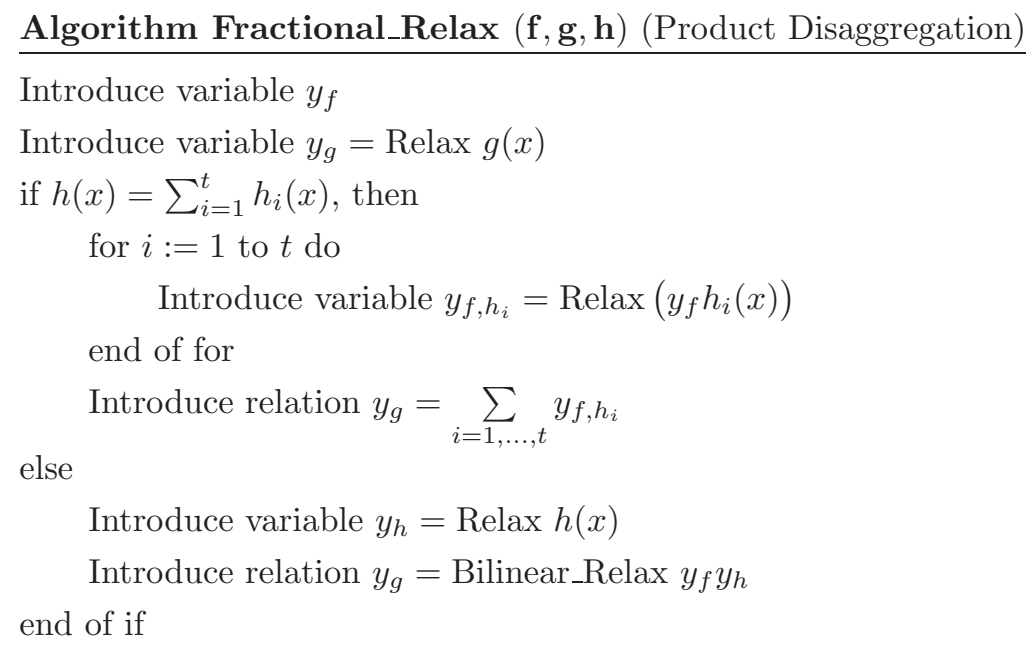

The bilinear term is relaxed using its convex and concave envelopes [26, 2].

Algorithm Bilinear_Relax $\mathbf{y}_{\mathbf{i}} \mathbf{y}_{\mathbf{j}}$ (Convex/Concave Envelope) 


$$
\begin{aligned}
& \text { Bilinear_Relax } y_{i} y_{j} \geq y_{i}^{u} y_{j}+y_{j}^{u} y_{i}-y_{i}^{u} y_{j}^{u} \\
& \text { Bilinear_Relax } y_{i} y_{j} \geq y_{i}^{l} y_{j}+y_{j}^{l} y_{i}-y_{i}^{l} y_{j}^{l} \\
& \text { Bilinear_Relax } y_{i} y_{j} \leq y_{i}^{u} y_{j}+y_{j}^{l} y_{i}-y_{i}^{u} y_{j}^{l} \\
& \text { Bilinear_Relax } y_{i} y_{j} \leq y_{i}^{l} y_{j}+y_{j}^{u} y_{i}-y_{i}^{l} y_{j}^{u}
\end{aligned}
$$

We are now left with the task of outer-estimating the univariate functions. At present, we do not attempt to detect individual functional properties, but decompose a univariate function as a recursive sum and product of monomial, logarithmic, and power terms. This keeps the implementation simple and accounts for most of the functions typically encountered in practical problems. More functions may be added to those listed above without much difficulty.

\section{Algorithm Univariate_Relax $\mathbf{f}\left(\mathbf{x}_{\mathbf{j}}\right)$ (Recursive Sums and Products)}

if $f\left(x_{j}\right)=c x_{j}^{p}$ then

Introduce variable $y_{f}=$ Monomial_Relax $c x_{j}^{p}$

else if $f\left(x_{j}\right)=c p^{x_{j}}$ then

Introduce variable $y_{f}=$ Power_Relax $c p^{x_{j}}$

else if $f\left(x_{j}\right)=c \log \left(x_{j}\right)$ then

Introduce variable $y_{f}=$ Logarithmic_Relax $c \log \left(x_{j}\right)$

else if $f\left(x_{j}\right)=\prod_{i=1}^{t} f_{i}\left(x_{j}\right)$, then

for $i:=1$ to $t$ do

Introduce variable $y_{f_{i}}$, such that $y_{f_{i}}=\operatorname{Relax} f_{i}(x)$

end of for

Introduce variable $y_{f}$, such that $y_{f}=$ Multilinear_Relax $\prod_{i=1}^{t} y_{f_{i}}$

else if $f\left(x_{j}\right)=\sum_{i=1}^{t} f_{i}\left(x_{j}\right)$, then

for $i:=1$ to $t$ do

Introduce variable $y_{f_{i}}$, such that $y_{f_{i}}=\operatorname{Relax} f_{i}\left(x_{j}\right)$

end of for

Introduce variable $y_{f}$, such that $y_{f}=\sum_{i=1}^{t} y_{f_{i}}$

end of if

We do not detail each of the procedures Monomial_Relax, Power_Relax and Logarithmic_Relax, as they may be easily derived by constructing the corresponding convex/concave envelopes over $\left[x_{j}^{l}, x_{j}^{u}\right]$. We do, however, address some common characteristic problems that arise in the derivation of these envelopes. 


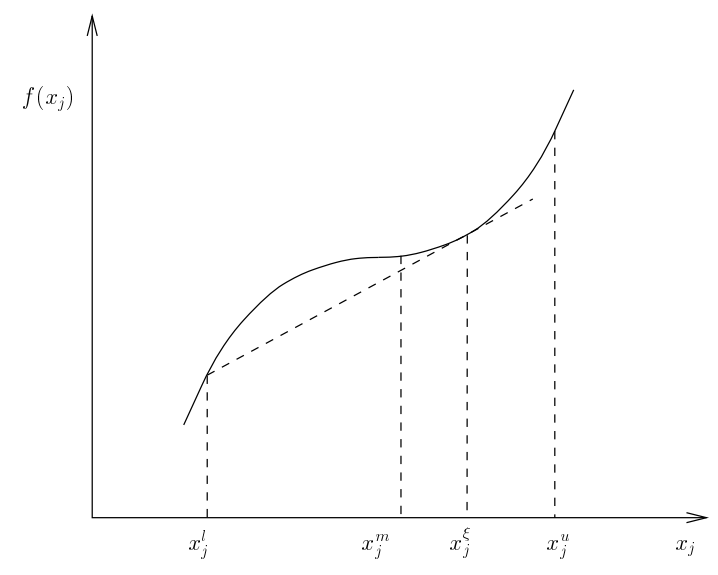

Figure 1: Developing the convex envelope for concavoconvex functions

\subsection{Concavoconvex Function}

A univariate function $f\left(x_{j}\right)$ is concavoconvex over an interval $\left[x_{j}^{l}, x_{j}^{u}\right]$ if, for some $x_{j}^{m}, f\left(x_{j}\right)$ is concave over $\left[x_{j}^{l}, x_{j}^{m}\right]$ and is convex over $\left[x_{j}^{m}, x_{j}^{u}\right]$. We define:

$$
\check{f}\left(x_{j}\right)= \begin{cases}f\left(x_{j}\right) & x_{j} \in\left[x_{j}^{m}, x_{j}^{u}\right] \\ +\infty & \text { otherwise. }\end{cases}
$$

We are interested in locating a point $x_{j}^{\xi}$, with a subdifferential $x^{*}$ to $f\left(x_{j}\right)$ at $x_{j}^{\xi}$ such that the corresponding tangent hyperplane passes through $\left(x_{j}^{l}, f\left(x_{j}^{l}\right)\right)$ (see Figure 1). We denote the conjugate of a function $f$ by $f^{*}$ and the subgradient set of $f$ at $x$ by $\partial f(x)$. Given $\bar{x}$, we define $g_{\bar{x}}(x)=\check{f}(x+\bar{x})$. Notice that $x_{j}^{\xi}$ may not be unique. Given $(\bar{x}, \bar{y})$, we consider the problem of identifying the set $X$ of all $x_{j}^{\xi}$ such that there exists a subdifferential, $x^{*}$, of $\check{f}\left(i . e ., x^{*} \in \partial \check{f}\left(x_{j}^{\xi}\right)\right)$ and $g_{\bar{x}}^{*}\left(x^{*}\right)=\bar{y}$. If $\bar{x}<x_{j}$ for all $x_{j} \in\left[x_{j}^{m}, x_{j}^{u}\right]$, then $x \in \partial g_{\bar{x}}^{*}\left(x^{*}\right)>0$. It follows that $g_{\bar{x}}^{*}\left(x^{*}\right)$ is a strictly increasing function of $x^{*}$. Therefore, the inverse $g_{\bar{x}}^{*-1}$ is unique. Inverting, we get $x^{*}=g_{\bar{x}}^{*-1}(\bar{y})$. Since $\partial g_{\bar{x}}^{*}=\left(\partial g_{\bar{x}}\right)^{-1}$,

$$
X=\left(\partial \check{f}^{*}\right)\left(g_{x_{j}^{l}}^{*-1}\left(f\left(x_{j}^{l}\right)\right)\right)=\left(\partial \check{f}^{*}\right)\left(\left(\check{f}^{*}-\cdot x_{j}^{l}\right)^{-1}\left(f\left(x_{j}^{l}\right)\right)\right) .
$$

When $f(x)$ is differentiable, it may be simpler to use the gradient expression:

$$
f\left(x_{j}^{l}\right)-f\left(x_{j}\right)+\nabla f\left(x_{j}\right)\left(x_{j}^{l}-x_{j}\right)=0 .
$$

Note that the left-hand-side of the above equation is an increasing function of $x_{j}$ as long as $x_{j} \geq x_{j}^{l}$ and, hence, any numerical technique like the secant method or bisection method may be used for its solution.

Similar techniques may be used to derive the concave envelope of a concavoconvex function, and the envelopes of a convexoconcave function. 


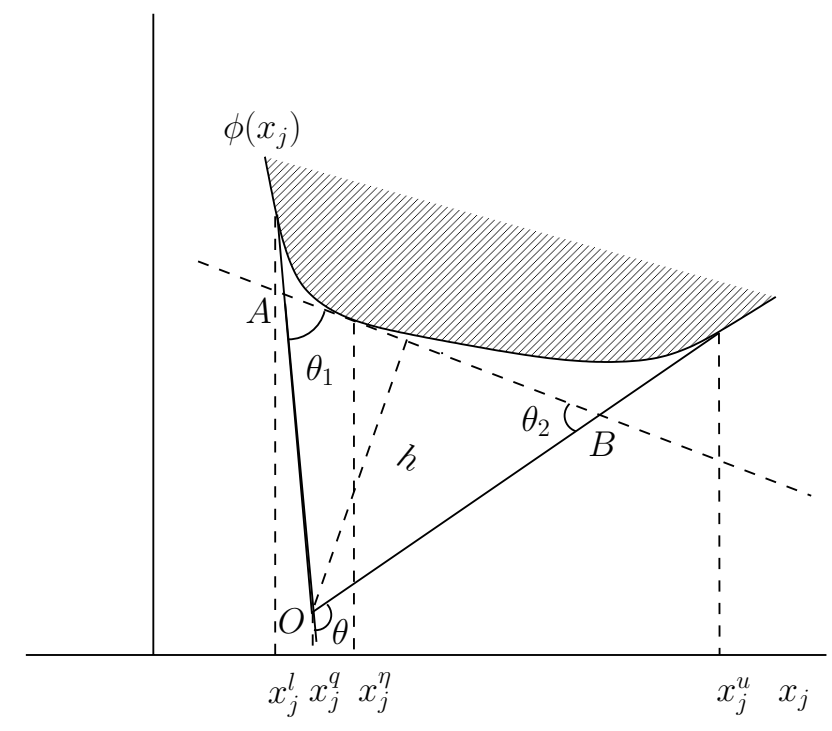

Figure 2: Outer-approximation of convex functions

\subsection{Outer-Approximation}

The relaxations to factorable programs derived above are typically nonlinear. However, nonlinear programs are harder to solve and are associated with more numerical issues than linear programs. For this reason, we propose the polyhedral outer-approximation of the above-derived nonlinear relaxations, thus generating an entirely linear programming based relaxation.

Consider a convex function $g(x)$ and the inequality $g(x) \leq 0$. With $s \in$ $\partial g(\bar{x})$, we have the subgradient inequality:

$$
0 \geq g(x) \geq g(\bar{x})+s(x-\bar{x}) .
$$

We are mainly interested in outer-approximating univariate convex functions that lead to the inequality: $\phi\left(x_{j}\right)-y \leq 0$ (see Figure 2). Then, (1) reduces to:

$$
0 \geq \phi\left(x_{j}\right)-y \geq \phi\left(\bar{x}_{j}\right)+s\left(x_{j}-\bar{x}_{j}\right)-y .
$$

To construct a polyhedral outer-approximation, we need to locate points $x_{j}$ in $\left[x_{j}^{l}, x_{j}^{u}\right]$ such that the subgradient inequalities approximate $\phi\left(x_{j}\right)$ closely.

The problem of outer-approximating convex functions is closely related to that of polyhedral approximations to convex sets $[15,14]$. It is known that the distance between a planar convex figure and its best approximating $n$-gon is $O\left(1 / n^{2}\right)$ under various error measures like Hausdorff, and area of the symmetric difference. For convex functions, with vertical distance as the error measure, the convergence rate of the error of the approximation can be shown to be, at best, $O\left(1 / n^{2}\right)$ by considering a parabola $y=x^{2}[29]$. 


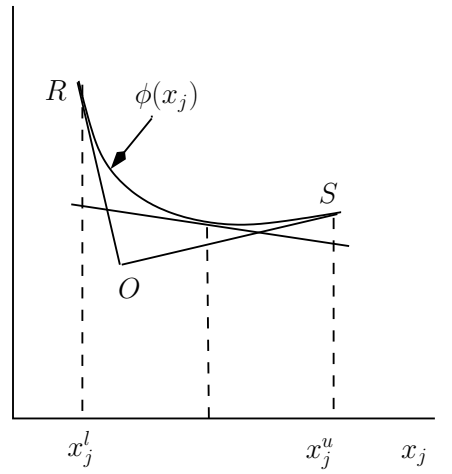

(a) Interval bisection

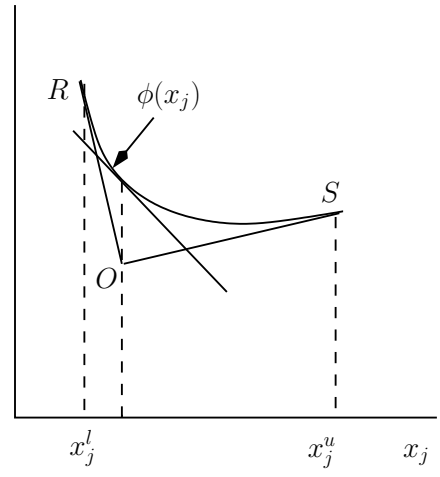

(c) Maximum error rule

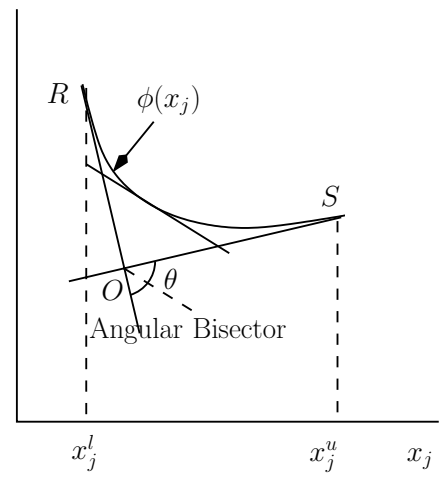

(e) Angle bisection

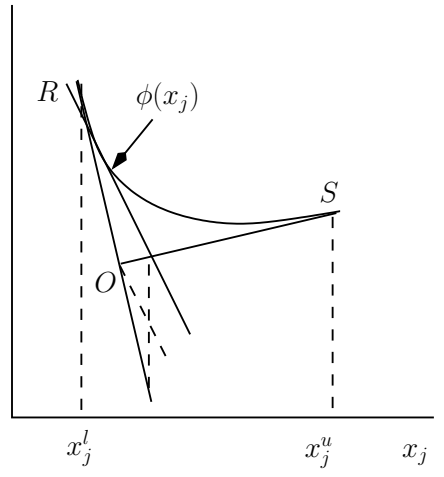

(b) Slope Bisection

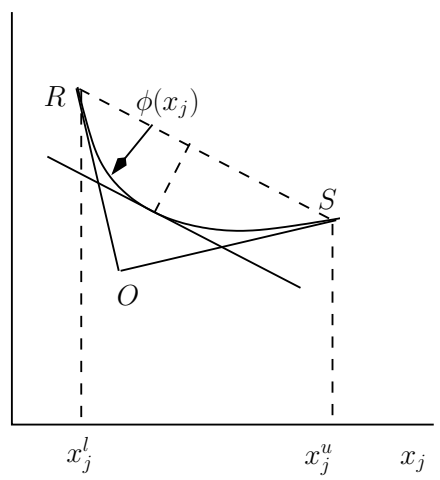

(d) Chord rule

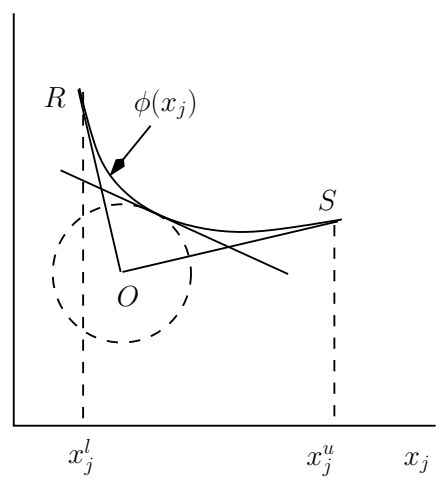

(f) Maximum projective error

Figure 3: Rules for selecting supporting lines in sandwich algorithms 
The sandwich algorithm is a template of outer-approximation schemes that attains the above asymptotic performance guarantee in a variety of cases [13, 29]. At a given iteration, this algorithm begins with a number of points at which tangential outer-approximations of the convex function have been constructed. Then, at every iterative step, the algorithm identifies the interval with the maximum outer-approximation error and subdivides it at a suitably chosen point. Five known strategies for identifying such a point are as follows:

1. Interval bisection: bisect the chosen interval (see Figure 3(a)).

2. Slope bisection: find the supporting line with a slope that is the mean of the slopes at the end points (see Figure 3(b)).

3. Maximum error rule: Construct the supporting line at the $x$ - ordinate of the point of intersection of the supporting lines at the two end points (see Figure 3(c)).

4. Chord rule: Construct the supporting line with the slope of the linear overestimator of the function (see Figure 3(d)).

5. Angle bisection: Construct the supporting line with the slope of the angular bisector of the outer angle $\theta$ of $R O S$ (see Figure 3(e)).

It was shown in [29] that, if the vertical distance between the convex function and the outer-approximation is taken as the error measure, then the first four procedures lead to an optimal performance guarantee. If $x_{j}^{l *}$ is the right-hand derivative at $x_{j}^{l}$ and $x_{j}^{u *}$ is the left-hand derivative at $x_{j}^{u}$, then, for $n \geq 2$, interval and slope bisection schemes produce a largest vertical error $\epsilon$ such that:

$$
\epsilon \leq \frac{9\left(x_{j}^{u}-x_{j}^{l}\right)\left(x_{j}^{u *}-x_{j}^{l *}\right)}{8 n^{2}} .
$$

The maximum error and chord rules have a better performance guarantee of:

$$
\epsilon \leq \frac{\left(x_{j}^{u}-x_{j}^{l}\right)\left(x_{j}^{u *}-x_{j}^{l *}\right)}{n^{2}} .
$$

Note that, since the initial $x_{j}^{u *}-x_{j}^{l *}$ can be rather large, the sandwich algorithm may still lead to high approximation errors for low values of $n$.

Let $\mathrm{G}(f)$ denote the set of points $(x, y)$ such that $y=f(x)$. Projective error is another error measure which is commonly used to describe the efficiency of an approximation scheme. If $\phi^{o}\left(x_{j}\right)$ is the outer-approximation of $\phi\left(x_{j}\right)$, then

$$
\epsilon_{p}=\sup _{p^{\phi^{o}} \in \mathrm{G}\left(\phi^{o}\right)} \inf _{p^{\phi} \in \mathrm{G}(\phi)}\left\{\left\|p^{\phi}-p^{\phi^{o}}\right\|\right\} .
$$

It was shown in [13] that the projective error of the angular bisection scheme decreases quadratically. In particular, if the initial outer angle of the initial function is $\theta$, then:

$$
\epsilon_{p} \leq \frac{9 \theta\left(x_{j}^{u}-x_{j}^{l}\right)}{8(n-1)^{2}} .
$$


We suggest another scheme where the supporting line is drawn at the point in $\mathrm{G}(\phi)$ which is closest to the intersection point of the slopes at the two endpoints (see Figure 3(f)). We call this the projective error rule. We next show that the resulting sandwich algorithm exhibits quadratic convergence.

Lemma 3.3. If $a, b, c$, and $d$ are four positive numbers such that $a c=b d$, then $a c+b d \leq a d+b c$.

Proof. $a c=b d$ implies that $a \geq b$ if and only if $c \leq d$. Therefore, $(b-a)(c-d) \geq$ 0. Expanding, we obtain the desired inequality.

Theorem 3.4. Consider a convex function $\phi\left(x_{j}\right):\left[x_{j}^{l}, x_{j}^{u}\right] \mapsto \mathbb{R}$ and the outerapproximation of $\phi$ formed by the tangents at $x_{j}^{l}$ and $x_{j}^{u}$. Let $R=\left(x_{j}^{l}, \phi\left(x_{j}^{l}\right)\right)$ and $S=\left(x_{j}^{u}, \phi\left(x_{j}^{u}\right)\right)$. Assume that the tangents at $R$ and $S$ intersect at $O$ and let $\theta$ be $\pi-\angle R O S$, and $L$ be the sum of the lengths of $R O$ and $O S$. Let $k=L \theta / \epsilon_{p}$. Then, the algorithm needs at most

$$
N(k)= \begin{cases}0, & k \leq 4 \\ \lceil\sqrt{k}-2\rceil, & k>4\end{cases}
$$

supporting lines for the outer-approximation to approximate $\phi\left(x_{j}\right)$ within $\epsilon_{p}$.

Proof. We argue that the supremum in (2) is attained at $O$ (see Figure 4). Indeed, since $\phi\left(x_{j}\right)$ is a convex function, as we move from $R$ to $S$ the derivative of $\phi$ increases, implying that the length of the perpendicular between the point on the curve and $R O$ increases attaining its maximum at $A$. For any two points $X$ and $Y$ on the curve, we denote by $L_{X Y}, \theta_{X Y}$, and $W_{X Y}$ the length of the line segment joining $X$ and $Y$, the angle $\pi-\angle X O Y$, and $L_{X O}+L_{O Y}$, respectively.

If $\phi\left(x_{j}\right)$ is not approximated within $\epsilon_{p}$, then

$$
\epsilon_{p}<L_{A O}=L_{O_{1} A} \tan \theta_{R A}=L_{O_{1} O} \sin \theta_{R A} \leq L_{R O} \theta_{R A} .
$$

Similarly, $\epsilon_{p}<L_{O S} \theta_{A S}$.

We prove the result by induction on $W_{R S} \theta_{R S} / \epsilon_{p}$. For the base case:

$$
\begin{aligned}
W_{R S} \theta_{R S} & =\left(L_{R O}+L_{O S}\right)\left(\theta_{R A}+\theta_{A S}\right) \\
& \geq\left(L_{O_{1} O}+L_{O O_{2}}\right)\left(\sin \theta_{R A}+\sin \theta_{A S}\right) \\
& \geq 2\left(L_{O_{1} O} \sin \theta_{R A}+L_{O O_{2}} \sin \theta_{A S}\right) \\
& =4 L_{A O} .
\end{aligned}
$$

It follows that, if $W_{R S} \theta_{R S} \leq 4 \epsilon_{p}$, the curve is approximated within tolerance.

To prove the inductive step, we need to show that:

$$
\begin{gathered}
N\left(W_{R S} \theta_{R S} / \epsilon_{p}\right) \geq \max 1+N\left(W_{R A} \theta_{R A} / \epsilon_{p}\right)+N\left(W_{A S} \theta_{A S} / \epsilon_{p}\right) \\
\text { s.t. } W_{R A}=L_{R O_{1}}+L_{O_{1} A}
\end{gathered}
$$




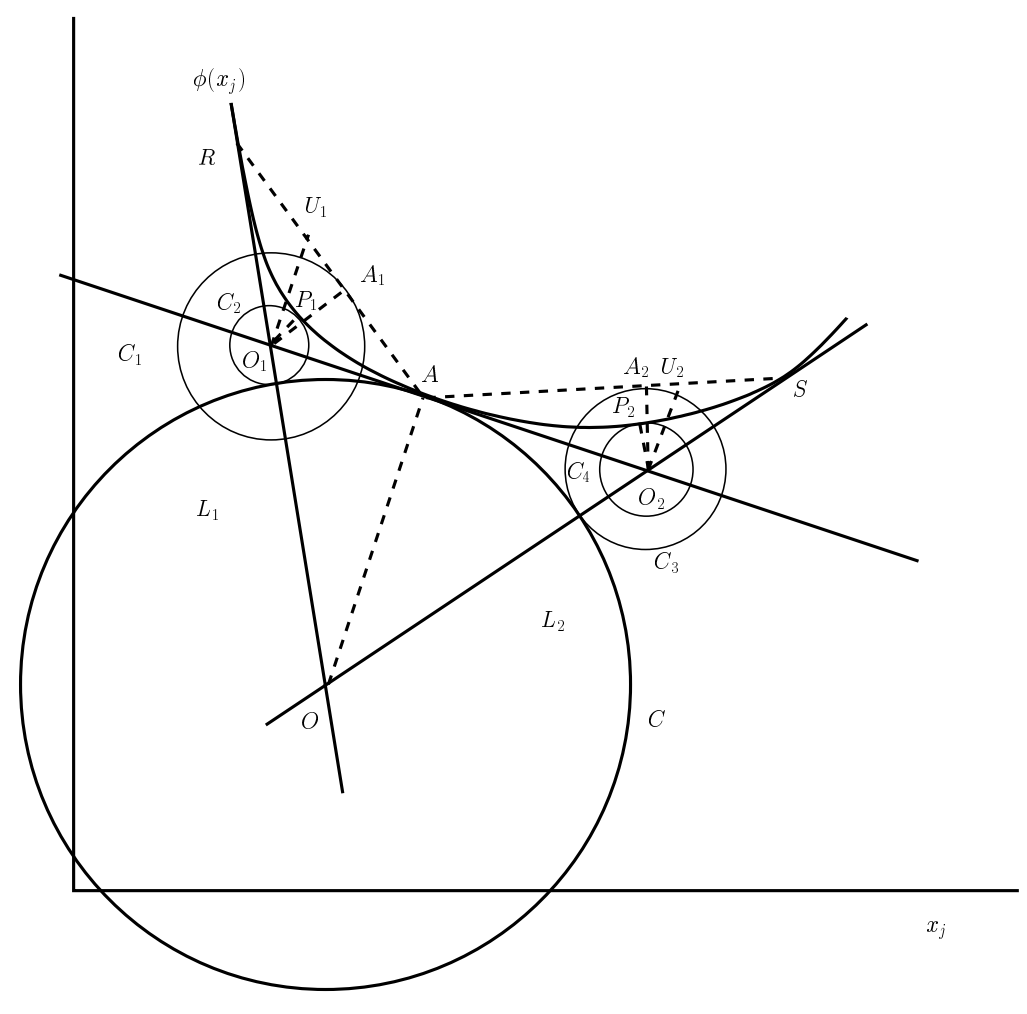

Figure 4: Proof of Theorem 3.4

$$
\begin{aligned}
& W_{A S}=L_{A O_{2}}+L_{O_{2} S} \\
& W_{R S}=L_{R O_{1}}+L_{O_{1} A} / \cos \theta_{R A}+L_{A O_{2}}+L_{O_{2} S} / \cos \theta_{A S} \\
& \theta_{R S}=\theta_{R A}+\theta_{A S} \\
& L_{O_{1} A} \tan \theta_{R A}>\epsilon_{p} \\
& L_{A O_{2}} \tan \theta_{A S}>\epsilon_{p} \\
& W_{R A}, W_{A S}, L_{R O_{1}}, L_{O_{1} A}, L_{A O_{2}}, L_{O_{2} S}, \theta_{R A}, \theta_{A S} \geq 0 .
\end{aligned}
$$

Since $N(\cdot)$ is an increasing function of (.), $W_{R A} \leq L_{R O_{1}}+L_{O_{1} A} / \cos \theta_{R A}=L_{R O}$, and $W_{A S} \leq L_{A O_{2}}+L_{O_{2} S} / \cos \theta_{A S}=L_{O S}$, we relax the above mathematical program by introducing variables $L_{R O}$ and $L_{O S}$ and prove the following tighter result:

$$
\begin{gathered}
N\left(W_{R S} \theta_{R S} / \epsilon_{p}\right) \geq \max 1+N\left(L_{R O} \theta_{R A} / \epsilon_{p}\right)+N\left(L_{O S} \theta_{A S} / \epsilon_{p}\right) \\
\text { s.t. } \theta_{R S}=\theta_{R A}+\theta_{A S} \\
W_{R S}=L_{R O}+L_{O S}
\end{gathered}
$$




$$
\begin{aligned}
& L_{R O} \theta_{R A}>\epsilon_{p} \\
& L_{O S} \theta_{A S}>\epsilon_{p} \\
& L_{R O}, L_{O S}, \theta_{R A}, \theta_{A S} \geq 0 .
\end{aligned}
$$

Since $\sqrt{L_{R O} \theta_{R A} / \epsilon_{p}} \geq 1$ and $\sqrt{L_{O S} \theta_{A S} / \epsilon_{p}} \geq 1$, we can apply the induction hypothesis. We only need to show that:

$$
\left\lceil\sqrt{W_{R S} \theta_{R S} / \epsilon_{p}}-2\right\rceil \geq 1+\max \left\{\left\lceil\sqrt{L_{R O} \theta_{R A} / \epsilon_{p}}-2\right\rceil+\left\lceil\sqrt{L_{O S} \theta_{A S} / \epsilon_{p}}-2\right\rceil\right\} .
$$

Since $\lceil c-2\rceil \geq 1+\lceil a-2\rceil+\lceil b-2\rceil$ when $c \geq a+b$, we prove the result by showing that:

$$
\sqrt{W_{R S} \theta_{R S} / \epsilon_{p}} \geq \max \left\{\sqrt{L_{R O} \theta_{R A} / \epsilon_{p}}+\sqrt{L_{O S} \theta_{A S} / \epsilon_{p}}\right\}
$$

where $L_{R O}+L_{O S}=W_{R S}$, and $\theta_{R A}+\theta_{A S}=\theta_{R S}$. Note that:

$$
\begin{aligned}
& \left(\sqrt{L_{R O} \theta_{R A}}+\sqrt{L_{O S} \theta_{A S}}\right)^{2} \\
\leq & \left(\sqrt{L_{R O} \theta_{R A}}+\sqrt{L_{O S} \theta_{A S}}\right)^{2}+\left(\sqrt{L_{R O} \theta_{A S}}-\sqrt{L_{O S} \theta_{R A}}\right)^{2} \\
= & \left(L_{R O}+L_{O S}\right)\left(\theta_{R A}+\theta_{A S}\right) \\
= & W_{R S} \theta_{R S} .
\end{aligned}
$$

Dividing by $\epsilon_{p}$ and taking the square root, we get:

$$
\sqrt{L_{R O} \theta_{R A} / \epsilon_{p}}+\sqrt{L_{O S} \theta_{A S} / \epsilon_{p}} \leq \sqrt{W_{R S} \theta_{R S} / \epsilon_{p}}
$$

Hence, the result is proven.

Implicit in the statement of the above theorem is the assumption that $\theta<\pi$ which is needed to ensure that the tangents at the end-points of the curve intersect. This is not a restrictive assumption since the curve can be segmented into two parts to ensure that the assumption holds.

The above result shows that the convergence of the approximation gap is quadratic in the number of points used for outer-approximation. This result can be extended to other error measures such as the area of the symmetric difference between the linear overestimator and the outer-approximation simply because the area of the symmetric difference grows super-linearly with the projective distance $h$. We provide a more in-depth analysis of the algorithm in [38].

Remark 3.5. Examples were constructed in [29] to show that there exist functions for which each of the above outer-approximation schemes based on interval, slope, and angle bisection, as well as the maximum and chord error rules perform arbitrarily worse compared to the others. We are not aware of any function for which the proposed projective error rule will be particularly bad. 


\section{Domain Reduction}

Domain reduction, also known as range reduction, is the process of cutting regions of the search space that do not contain an optimal solution. Various techniques for range reduction have been developed in $[41,18,17,20,30,31$, $35,43]$. We develop a theory of range reduction tools in this section and then derive earlier results in the light of these new developments.

\subsection{Theoretical Framework}

Consider a mathematical programming model:

$$
\begin{array}{ll}
\min & f(x) \\
\text { s.t. } & g(x) \leq b \\
& x \in X \subseteq \mathbb{R}^{n}
\end{array}
$$

where $f: \mathbb{R}^{n} \mapsto \mathbb{R}, g: \mathbb{R}^{n} \mapsto \mathbb{R}^{m}$, and $X$ denotes the set of "easy" constraints. The standard definition of the Lagrangian subproblem for $\mathrm{T}$ is:

$$
\inf _{x \in X} l(x, y)=\inf _{x \in X}\{f(x)-y(g(x)-b)\}, \text { where } y \leq 0 .
$$

Instead, we define the Lagrangian subproblem as:

$$
\inf _{x \in X} l^{\prime}\left(x, y_{0}, y\right)=\inf _{x \in X}\left\{-y_{0} f(x)-y g(x)\right\}, \text { where }\left(y_{0}, y\right) \leq 0,
$$

since the additional dual variable $y_{0}$ homogenizes the problem and allows us to provide a unified algorithmic treatment of range-reduction problems.

Assume that $b_{0}$ is an upper bound on the optimal objective function value of $\mathrm{T}$ and consider the following range-reduction problem:

$$
h^{*}=\inf _{x, u_{0}, u}\left\{h\left(u_{0}, u\right) \mid f(x) \leq u_{0} \leq b_{0}, g(x) \leq u \leq b, x \in X\right\},
$$

where $h$ is assumed to be a linear function. Problem (5) can be restated as:

$$
\begin{array}{rl}
\left(\mathrm{R}_{\mathrm{u}}^{\mathrm{C}}\right) \quad h^{*}=\inf _{x, u_{0}, u} & h\left(u_{0}, u\right) \\
\text { s.t. }- & y_{0}\left(f(x)-u_{0}\right)-y(g(x)-u) \leq 0 \quad \forall\left(y_{0}, y\right) \leq 0 \\
& \left(u_{0}, u\right) \leq\left(b_{0}, b\right), x \in X .
\end{array}
$$

Computing $h^{*}$ is as hard as solving T. Therefore, we lower bound $h^{*}$ with the optimal value of the following problem:

$$
\begin{array}{rl}
\left(\overline{\mathrm{R}_{\mathrm{u}}^{\mathrm{C}}}\right) \quad h_{L}=\inf _{u_{0}, u} & h\left(u_{0}, u\right) \\
\text { s.t. } & y_{0} u_{0}+y u+\inf _{x \in X}\left\{-y_{0} f(x)-y g(x)\right\} \leq 0 \quad \forall\left(y_{0}, y\right) \leq 0 \\
& \left(u_{0}, u\right) \leq\left(b_{0}, b\right) .
\end{array}
$$


Much insight into our definition of the domain reduction problem and its potential uses is gained by restricting $h\left(u_{0}, u\right)$ to $a_{0} u_{0}+a u$ where $\left(a_{0}, a\right) \geq 0$ and $\left(a_{0}, a\right) \neq 0$. Interesting applications arise when $\left(a_{0}, a\right)$ is set to one of the principal directions in $\mathbb{R}^{m+1}$.

Using Fenchel-Rockafellar duality, the following algorithm is derived in [38] to iteratively obtain lower and upper bounds on the range-reduction problem with a linear objective. The algorithm is similar to the iterative algorithm commonly used to solve the Lagrangian relaxation of $\mathrm{T}$. The treatment herein can be generalized to arbitrary lower semicontinuous functions $h\left(u, u_{0}\right)$. We refer the reader to [38] for a detailed discussion, related proofs, and generalizations.

\section{Algorithm SimpleReduce}

Step 0 . Set $K=0, u_{0}^{0}=b_{0}, u^{0}=b$.

Step 1. Solve the relaxed dual of (5):

$$
\begin{array}{rlr}
h_{U}^{K}=\max _{\left(y_{0}, y\right)} & \left(y_{0}+a_{0}\right) b_{0}+(y+a) b-z & \\
\text { s.t. } & z \geq y_{0} u_{0}^{k}+y u^{k} \quad k=0, \ldots, K-1 \\
& \left(y_{0}, y\right) \leq-\left(a_{0}, a\right) &
\end{array}
$$

Let the solution be $\left(y_{0}^{K}, y^{K}\right)$.

Step 2. Solve the Lagrangian subproblem:

$$
\begin{aligned}
\inf _{x} l^{\prime}\left(x, y_{0}^{K}, y^{K}\right)=-\max _{x, u_{0}, u} & y_{0}^{K} u_{0}+y^{K} u \\
\text { s.t. } \quad & f(x) \leq u_{0} \\
& g(x) \leq u \\
& x \in X
\end{aligned}
$$

Let the solution be $\left(x^{K}, u_{0}^{K}, u^{K}\right)$. Note that $\left(y_{0}, y\right) \leq 0$ implies that $u_{0}^{K}=f\left(x^{K}\right)$ and $u^{K}=g\left(x^{K}\right)$.

Step 3. Augment and solve the relaxed primal problem:

$$
\begin{aligned}
h_{L}^{K}=\min _{\left(u_{0}, u\right)} & a_{0} u_{0}+a u \\
\text { s.t. } & y_{0}^{k} u_{0}+y^{k} u+\inf _{x} l^{\prime}\left(x, y_{0}^{k}, y^{k}\right) \leq 0 \\
& \quad k=1, \ldots, K \\
& \left(u_{0}, u\right) \leq\left(b_{0}, b\right)
\end{aligned}
$$

Step 4. Termination check: If $h_{U}^{K}-h_{L}^{K} \leq$ tolerance, stop; otherwise, set $K=K+1$ and goto Step 1 .

The reader will notice that the Algorithm SimpleReduce reduces to the standard Lagrangian lower bounding procedure for problem $\mathrm{T}$ when $\left(a_{0}, a\right)=(1,0)$. In this case, $y_{0}$ can be fixed to -1 and the relaxed master problem is equivalent to maintaining the maximum bound obtained from the solved Lagrangian 
subproblems: $\max _{k=1, \ldots, K} \inf _{x \in X} l(x, y)$. Similarly, Algorithm SimpleReduce reduces to the standard Lagrangian lower bounding procedure for the problem:

$$
\begin{aligned}
\left(\mathrm{R}_{\mathrm{g}_{\mathrm{i}}}\right) \quad \min \quad & g_{i}(x) \\
\text { s.t. } & g(x) \leq b \\
& f(x) \leq b_{0} \\
& x \in X \subseteq \mathbb{R}^{n}
\end{aligned}
$$

when $a_{i}=1$ and $a_{j}=0$ for all $j \neq i$. Since the Lagrangian subproblem is independent of $\left(a_{0}, a\right)$ and homogenous in $\left(y_{0}, y\right)$, the cuts $z \geq y_{0} u_{0}^{k}+y u^{k}$ and $y_{0}^{k} u_{0}+y^{k} u+\inf _{x} l\left(x, y_{0}^{k}, y^{k}\right) \leq 0$ derived during the solution of the Lagrangian relaxation at any node in a branch-and-bound tree can be used to hot-start the Lagrangian procedure that determines lower bounds for $g_{i}(x)$ as long as $\left(y_{0}^{k}, y^{k}\right)$ can be scaled to be less than or equal to $\left(a_{0}, a\right)$. Note that it may not always be possible to solve the Lagrangian subproblem efficiently, which may itself be NP-hard. In such cases, any suitable relaxation of the Lagrangian subproblem can be used instead to derive a weaker lower bound on $h\left(u_{0}, u\right)$.

\subsection{Applications to Polyhedral Sets}

We shall, as an example, specialize some of the results of the previous section to polyhedral sets using linear programming duality theory. Consider the following dual linear programs:

$$
\begin{aligned}
& \text { (PP) } \min c x \\
& \text { s.t. } A x \leq b \quad \stackrel{\text { Duality }}{\Longleftrightarrow} \\
& \text { (PD) } \max \lambda b \\
& \text { s.t. } \lambda A=c \\
& \lambda \leq 0
\end{aligned}
$$

where $A \in \mathbb{R}^{n \times m}$. Construct the following perturbation problem and its dual:

$$
\begin{aligned}
& \text { (PU) } \min u_{i} \\
& \text { (PR) } \max r b+s U \\
& \text { s.t. } A x \leq b+e_{i} u_{i} \quad \stackrel{\text { Duality }}{\Longleftrightarrow} \\
& \text { s.t. } \quad r A+s c=0 \\
& c x \leq U \\
& -r e_{i}=1 \\
& r, s \leq 0 \text {. }
\end{aligned}
$$

Note that the optimal objective function value of PU provides a lower bound for $A_{i} x-b_{i}$ assuming that $U$ is an upper bound on $c x$. In particular, PU is the range-reduction problem defined in (5) when $h\left(u_{0}, u\right)=u_{i}$. Then, PR models the dual of PU which is equivalent to the following Lagrangian relaxation:

$$
\max _{y \geq 0, y_{i}=1} \min _{x \in X} y(A x-b)+b_{i}+y_{0}(c x-U)
$$

In this case, $l^{\prime}\left(x, y_{0}^{k}, y^{k}\right)=y_{0} c x+y A x$ and moving the terms independent of $x$ outside the inner minimization we obtain the Lagrangian relaxation:

$$
\max _{y \geq 0, y_{i}=1}-y_{0} U-y b+b_{i}+\min _{x \in X} y_{0} c x+y A x
$$


Algorithm SimpleReduce then reduces to the outer-approximation procedure to solve the Lagrangian dual (see [4]). Let $(\bar{r}, \bar{s})$ be a feasible solution to the above problem. Then, either $s=0$ or $s<0$.

- Case 1: $s<0$. The set of solutions to (PR) are in one-to-one correspondence with the solutions to (PD) by the following relation:

$$
s=1 / \lambda_{i}, \quad r=-\lambda s .
$$

- Case 2: $s=0$.

$$
\begin{aligned}
& \text { (PRM) } \max r b \\
& \begin{aligned}
\text { s.t. } r A=0 \quad \text { Duality } & \text { s.t. } A x \leq b+e_{i} u_{i} . \\
-r e_{i} & =1
\end{aligned} \\
& r \leq 0
\end{aligned}
$$

The dual solutions are in direct correspondence with those of (PUM).

Note that PR and PD both arise from the same polyhedral cone:

$$
\begin{aligned}
& r A+s c=0 \\
& r, s \leq 0
\end{aligned}
$$

with different normalizing planes $s=-1$ and $-r e_{i}=1$. Therefore, apart from the hyperplane at infinity, the correspondence between both sets is one-to-one. As a result, dual solutions of PP (not necessarily optimal) can be used to derive lower bounds for PU. The dual solutions are useful in tightening bounds on the variables when some or all of the variables are restricted to be integer. Also, when the model includes additional nonlinear relations, the domain-reduction is particularly useful since relaxation schemes for nonlinear expressions often utilize variable bounds as was done in Section 3.

\subsection{Relation to Earlier Works}

\subsubsection{Optimality-Based Range Reduction}

The following result is derived as a simple corollary of Algorithm SimpleReduce.

Theorem 4.1. Suppose the Lagrangian subproblem in (4) is solved for a certain dual multiplier vector $y \leq 0$. Then, for each $i$ such that $y_{i} \neq 0$, the cut $g_{i}(x) \geq$ $\left(b_{0}-\inf _{x} l(x, y)\right) / y_{i}$ does not chop off any optimal solution of $T$.

Proof. A lower bound on $g_{i}(x)$ is obtained by solving the relaxed primal problem in Step 3 of Algorithm SimpleReduce with $\left(a_{0}, a\right)=-e_{i}$, where $e_{i}$ is the $i^{\text {th }}$ column of the identity matrix. It is easily seen that the optimal solution sets $u_{j}=b_{j}$ for $j \neq i$ and the optimal solution value is $\left(b_{0}-\inf _{x} l(x, y)\right) / y_{i}$. 
We show next that simple applications of the above result produce the rangereduction schemes of $[31,43]$. Assuming that appropriate constraint qualifications hold, the strong duality theorem of convex programming asserts that the optimal objective function value, $L$, of a convex relaxation with optimal dual multipliers, $y^{*}$, is less than or equal to the optimal value of the corresponding Lagrangian subproblem, $\inf _{x} l\left(x, y^{*}\right)$. The cut

$$
g_{i}(x) \geq(U-L) / y_{i}^{*}
$$

proposed in Theorem 2 of [31] is therefore either the same as derived in Theorem 4.1 or weaker than it. Theorem 3 and Corollaries 1, 2, and 3 in [31] as well as the marginals-based reduction techniques in $[41,20]$ and the early integer programming literature [27] follow similarly. Also similarly, follows Corollary 4 in [31] which deals with probing, the process of temporarily fixing variables at their bounds and drawing inferences based on range reduction. More general probing procedures are developed in [38] as a consequence of the domain reduction strategy of Section 4.1.

In [43], a lower bound is derived on the optimal objective function of $\mathrm{T}$ by solving an auxiliary contraction problem. We now derive this result as a direct application of Theorem 4.1. Consider an auxiliary problem constrained by $f(x)-U \leq 0$ with an objective function $f_{a}(x)$, for which an upper bound $f_{a}^{u}$ is known. Let $l_{a}(x, y)$ be the Lagrangian function for the auxiliary problem. Consider a dual solution, $y_{a}$, with a positive dual multiplier, $y_{a f}$, corresponding to $f(x)-U \leq 0$. By Theorem 4.1, the following cut does not chop off an optimal solution:

$$
y_{a f}\{f(x)-U\} \leq f_{a}^{u}-\inf _{x} l_{a}(x, y) .
$$

Using the auxiliary contraction problem below:

$$
\begin{array}{cl}
\min & x_{i} \\
\text { s.t. } & g(x) \leq 0 \\
& f(x)-U \leq 0 \\
& x \in X \subseteq \mathbb{R}^{n},
\end{array}
$$

Theorem 3, Theorem 4, Corollary 1, and Corollary 2 of [43] follow immediately.

\subsubsection{Feasibility-Based Range Reduction}

Consider the constraint set $\sum_{j=1}^{n} a_{i j} x_{j} \leq b_{i}, i=1, \ldots, m$. Processing one constraint at a time, the following variable bounds are derived:

$$
\begin{cases}x_{h} \leq \frac{1}{a_{i h}}\left(b_{i}-\sum_{j \neq h} \min \left\{a_{i j} x_{j}^{u}, a_{i j} x_{j}^{l}\right\}\right), & a_{i h}>0 \\ x_{h} \geq \frac{1}{a_{i h}}\left(b_{i}-\sum_{j \neq h} \min \left\{a_{i j} x_{j}^{u}, a_{i j} x_{j}^{l}\right\}\right), & a_{i h}<0\end{cases}
$$


where $x_{j}^{u}$ and $x_{j}^{l}$ are the tightest upper and lower bounds available for $x_{j}$ at the current node. This procedure was referred to as "feasibility-based range reduction" in [35] and has been used extensively in mixed-integer linear programming (cf. [34]). We now derive this scheme as a consequence of the duality framework of Section 4.1. Note that this feasibility-based range reduction may be viewed as a rather simple application of Fourier-Motzkin elimination to the following problem-obtained by relaxing all but the $i^{\text {th }}$ constraint:

$$
\begin{array}{ll}
\min & x_{h} \\
\text { s.t. } & a_{i} x \leq b_{i} \\
& x \leq x^{u} \\
& x \geq x^{l} .
\end{array}
$$

Assuming $a_{i h}<0$ and $x_{h}$ is at not at its lower bound, the optimal dual solution can easily be gleaned from the above linear program to be:

$$
\begin{aligned}
& y=1 / a_{i h} \\
& r_{j}=-\max \left\{a_{i j} / a_{i h}, 0\right\} \text { for all } j \neq h \\
& s_{j}=\min \left\{a_{i j} / a_{i h}, 0\right\} \text { for all } j \neq h \\
& r_{h}=s_{h}=0
\end{aligned}
$$

where $y, r_{j}$, and $s_{j}$ are the dual multipliers corresponding to $a_{i} x \leq b_{i}, x_{j} \leq x_{j}^{u}$, and $x_{j} \geq x_{j}^{l}$, respectively. Then, the following relaxed primal master problem is constructed:

$$
\min \left\{x_{h} \mid-x_{h}+y u+r v-s w \leq 0, u \leq b, v \leq x^{u}, w \geq x^{l}\right\} .
$$

Consequently, the bounds presented in (6) follow easily from (7).

\subsection{New Range Reduction Procedures}

\subsubsection{Duality-based Reduction}

When a relaxation is solved by dual ascent procedures such as dual Simplex or Lagrangian relaxations, dual feasible solutions are generated at every iteration of the algorithm. These solutions can be maintained in a set, $S$, of solutions feasible to the following system:

$$
\begin{aligned}
& r A+s c=0 \\
& r, s \leq 0
\end{aligned}
$$

As described in Section 4.2, the feasible solutions to PD as well as PR belong to the polyhedral cone defined by the above inequalities. Whenever a variable bound is updated as a result of partitioning or otherwise, or a new upper bounding solution is found for the problem, new bounds on all problem variables 
are obtained by evaluating the objective function of $\mathrm{PR}$ - with the appropriate perturbation-using the dual solutions stored in $S$.

In fact, if the matrix $A$ also depends on the bounds of the variables as is the case when nonlinear programs are relaxed to linear outer-approximations (see Section 3) then a slightly cleverer scheme can be employed by treating the bounds as "easy" constraints and including them in the Lagrangian subproblem. Consider, for example, a linear relaxation which takes the following form:

$$
\begin{array}{ll}
\min & c\left(x^{L}, x^{U}\right) x \\
\text { s.t. } & l\left(x^{L}, x^{U}\right) \leq A\left(x^{L}, x^{U}\right) x \leq u\left(x^{L}, x^{U}\right) \\
& x^{L} \leq x \leq x^{U}
\end{array}
$$

Assume that (8) contains the objective function cut $c\left(x^{L}, x^{U}\right) x \leq U$. Construct a matrix $\Pi$, whose each row is a vector of dual multipliers of (8) (possibly from the set $S$ described above). Feasibility-based tightening is done on the surrogate constraints generated by pre-multiplying the linear constraint set with this $\Pi$ matrix. Clearly, there exists a vector $\pi$ of dual multipliers that proves the best bound which can be derived from the above relaxation. The feasibility-based range reduction is a special case of duality-based tightening when $\Pi$ is chosen to be the identity matrix. The marginals-based range reduction is a special case of the proposed duality-based range-reduction where the optimal value dual solution is augmented with -1 as the multiplier for the objective function cut.

\subsubsection{Learning Reduction Heuristic}

In order to guarantee exhaustiveness, most rectangular partitioning schemes resort to periodic bisection of the feasible space along each variable axis. Let us assume that, after branching, one of the child nodes turns out to be inferior. Then, it is highly probable that a larger region could have been fathomed if a proper branching point was chosen initially. In cases of this sort, a learning reduction procedure can introduce an error-correcting capability by expanding the region that is provably inferior. In particular, consider a node $N$ where the following relaxation is generated:

$$
\begin{array}{cl}
\min _{x} & \left(f_{N}\left(x^{L}, x^{U}\right)\right)(x) \\
\text { s.t. } & \left(g_{N}\left(x^{L}, x^{U}\right)\right)(x) \leq 0 \\
& x^{L} \leq x \leq x^{U}
\end{array}
$$

where $\left(f_{N}\left(x^{L}, x^{U}\right)\right)(\cdot)$ and $\left(g_{N}\left(x^{L}, x^{U}\right)\right)(\cdot)$ are convex functions. We assume that the relaxation strategy is such that, when a variable is at its bound, the dependent functions are represented exactly. Consider now the partitioning of node $N$ into $N_{1}$ and $N_{2}$ by branching along $x_{j}$ at $x_{j}^{B}$. Define

$$
x^{a}=\left[x_{1}^{U}, \ldots, x_{j-1}^{U}, x_{j}^{B}, x_{j+1}^{U}, \ldots, x_{n}^{U}\right]
$$


and

$$
x^{b}=\left[x_{1}^{L}, \ldots, x_{j-1}^{L}, x_{j}^{B}, x_{j+1}^{L}, \ldots, x_{n}^{L}\right] .
$$

$N_{1}$ and $N_{2}$ are constructed so that $x^{L} \leq x \leq x^{a}$ for all $x \in N_{1}$ and $x^{b} \leq x \leq x^{U}$ for all $x \in N_{2}$. Assume further that node $N_{1}$ is found to be inferior and $y^{*}$ is the vector of optimal dual multipliers using the relaxation generated at node $N_{1}$. The constraint $x \leq x_{j}^{B}$ is assumed to be active in the solution of $N_{1}$.

If we can construct the optimal dual solution $y_{N_{2}^{P}}^{*}$ for the following program:

$$
\begin{array}{cl}
\min _{x} & \left(f_{N_{2}}\left(x^{b}, x^{U}\right)\right)(x) \\
\text { s.t. } & \left(g_{N_{2}}\left(x^{b}, x^{U}\right)\right)(x) \leq 0 \\
& x^{b} \leq x \leq x^{U} \\
& x_{j} \geq x_{j}^{B},
\end{array}
$$

then we can apply the techniques developed in earlier sections for domain reduction. Construction of $y_{N_{2}^{P}}^{*}$ is expensive since it involves lower bounding the nonconvex problem at $N_{2}$. However, when the relaxation technique has certain properties, $y_{N_{2}^{P}}^{*}$ can be computed efficiently. We assumed that the relaxations at nodes $N_{1}$ and $N_{2}$ are identical when $x_{j}=x_{j}^{B}$ and that $x \leq x_{j}^{B}$ is active. These assumptions imply that not only is the optimal solution of the relaxation at $N_{1}$ optimal to $\mathrm{P}_{\mathrm{N}_{2}}$, but the geometry of the KKT conditions at optimality is identical apart from the activity of the constraint $x_{j} \leq x_{j}^{B}$. Therefore, it is possible to identify the set of active constraints for $\mathrm{P}_{\mathrm{N}_{2}}$. Once this is done, $y_{N_{2}^{P}}^{*}$ can be computed by solving a set of linear equations. In fact, the only change required to $y_{N_{1}}^{*}$ is in the multiplier corresponding to $x_{j} \leq x_{j}^{B}$.

There is, however, a subtle point that must be realized while identifying the set of active constraints. A function $r(x)$ in the original nonconvex program may be relaxed to $\max \left\{r_{1}(x), \ldots, r_{k}(x)\right\}$ where each $r_{i}(\cdot)$ is separable and convex. In this case, the function attaining the supremum can change between the nodes $N_{1}$ and $N_{2}$. We illustrate this through the example of the epigraph of a bilinear function $z \leq x_{j} w$. We relax $z$ using the bilinear envelopes as follows [25]:

$$
z \leq \min \left\{x_{j}^{L} w+w^{U} x_{j}-x_{j}^{L} w^{U}, x_{j}^{U} w+w^{L} x_{j}-x_{j}^{U} w^{L}\right\} .
$$

In particular, at node $N_{1}$ :

$$
z \leq \min \left\{x_{j}^{L} w+w^{U} x_{j}-x_{j}^{L} w^{U}, x_{j}^{B} w+w^{L} x_{j}-x_{j}^{B} w^{L}\right\}
$$

and at node $N_{2}$ :

$$
z \leq \min \left\{x_{j}^{B} w+w^{U} x_{j}-x_{j}^{B} w^{U}, x_{j}^{U} w+w^{L} x_{j}-x_{j}^{U} w^{L}\right\} .
$$

When $x=x_{j}^{B}$ in the relaxation at node $N_{1}$, the dominating constraint is $z \leq$ $x_{j}^{B} w+w^{L} x_{j}-x_{j}^{B} w^{L}$ whereas, in $\mathrm{P}_{\mathrm{N}_{2}}$, the dominating constraint is $z \leq x_{j}^{B} w+$ $w^{U} x_{j}-x_{j}^{B} w^{U}$. If $\mu$ is the dual multiplier for constraint $z \leq x_{j}^{B} w+w^{L} x_{j}-x_{j}^{B} w^{L}$ 
in $y_{N_{1}}^{*}$, then $\mu$ should be the dual multiplier of $z \leq x_{j}^{B} w+w^{U} x_{j}-x_{j}^{B} w^{U}$ in $y_{N_{2}^{P}}^{*}$. Adding $\left(w^{U}-w^{L}\right)\left(x_{j}^{B}-x_{j}\right) \geq 0$, yields the desired constraint:

$$
z \leq x_{j}^{B} w+w^{L} x_{j}-x_{j}^{B} w^{L} .
$$

Thus, it is possible that the multiplier corresponding to the upper bounding constraint $x_{j} \leq x_{j}^{B}$ increases when a dual solution is constructed for $\mathrm{P}_{\mathrm{N}_{2}}$ from a dual solution of $N_{1}$. Such an increase would not occur if the only difference between the relaxations at $N_{1}$ and $N_{2}$ is the bound on $x_{j}$, which is most often the case with relaxations of integer linear programs.

\section{$5 \quad$ Node Partitioning Schemes}

Rectangular partitioning - to which we restrict our attention - splits the feasible region, at every branch-and-bound iteration, into two parts by intersecting it with the half-spaces of a hyperplane orthogonal to one of the coordinate axes. In order to define this hyperplane uniquely, the coordinate axis perpendicular to the hyperplane and the intercept of the hyperplane on the axis must be specified. The choice of the coordinate axis and the intercept are referred to as the branching variable and branching point selection, respectively.

\subsection{Branching Variable Selection}

The selection of the branching variable determines, to a large extent, the structure of the tree explored and affects the performance of a branch-and-bound algorithm significantly. We determine the branching variable through "violation transfer." First, violations of nonconvexities by the current relaxation solution are assigned to problem variables. Then, the violations are transferred between variables utilizing the functional relationships in the constraints and objective. Finally, violations are weighted to account for branching priorities and the potential relaxation improvement after branching, and a variable leading to the maximum weighted violation is chosen for branching.

Algorithm Relax of Section 3 reformulates the general problem such that multi-dimensional functions are replaced with either univariate functions or bilinear functions. We provide branching rules for both cases in this section.

Consider a variable $y$ that depends on $x_{j}$ through the relation $y=f\left(x_{j}\right)$. Let $\left(x_{j}^{*}, y^{*}\right)$ denote the value attained by $\left(x_{j}, y\right)$ in the relaxation solution. Define $X_{j}^{y}=f^{-1}\left(y^{*}\right)$. The violation of $x_{j}$ is informally defined to be the length of the interval obtained as the intersection of $\left[x_{j}^{L}, x_{j}^{U}\right]$ and the smallest interval around $x_{j}^{*}$ which contains, for every variable $y$ dependent on $x_{j}^{*}$, an $x_{j}^{y} \in X_{j}^{y}$.

The violations on all variables are computed in a two-phase process, the forward-transfer phase and the backward-transfer phase. In the forward transfer, the violations on the dependent variables are computed using the relaxation solution values for the independent variables using all the introduced relations. In the backward-transfer phase, these violations are transmitted back to the 
independent variables. For each dependent variable $y$ we derive an interval $\left[y^{l v}, y^{u v}\right]$. Similarly, we derive an interval $\left[x_{j}^{l v}, x_{j}^{u v}\right]$ for $x_{j}$.

\section{Algorithm ViolationTransfer}

Step 0. Set $y^{l v}=y^{u v}=y^{*}$. If $x_{j}$ is not required to be integer-valued, set $x_{j}^{l v}=x^{u v}=x_{j}^{*}$. Otherwise, set $x_{j}^{l v}=\left\lfloor x_{j}^{*}\right\rfloor$ and $x_{j}^{u v}=\left\lceil x_{j}^{*}\right\rceil$.

Step 1. Forward transfer:

$$
\begin{aligned}
& y^{l v}=\max \left\{y^{L}, \min \left\{y^{l v}, f\left(x_{j}^{l v}\right), f\left(x_{j}^{u v}\right)\right\}\right\} \\
& y^{u v}=\min \left\{y^{U}, \max \left\{f\left(x_{j}^{l v}\right), f\left(x_{j}^{u v}\right), y^{u v}\right\}\right\}
\end{aligned}
$$

Step 2. Backward transfer:

$$
\begin{aligned}
& x_{j}^{l v}=\max \left\{x_{j}^{L}, \min \left\{x_{j}^{l v}, f^{-1}\left(y^{l v}\right), f^{-1}\left(y^{u v}\right)\right\}\right\} \\
& x_{j}^{u v}=\min \left\{x_{j}^{U}, \max \left\{f^{-1}\left(y^{l v}\right), f^{-1}\left(y^{u v}\right), x_{j}^{u v}\right\}\right\}
\end{aligned}
$$

Step 3. $v_{y}=y^{u v}-y^{l v}, v_{x_{j}}=x_{j}^{u v}-x_{j}^{l v}$.

For bilinear functions, the violation transfer scheme is more involved. We refer the reader to [38] for details.

Define

$$
x^{l}=\left[x_{1}^{*}, \ldots, x_{j-1}^{*}, x_{j}^{l v}, x_{j+1}^{*}, \ldots, x_{n}^{*}\right]
$$

and

$$
x^{u}=\left[x_{1}^{*}, \ldots, x_{j-1}^{*}, x_{j}^{u v}, x_{j+1}^{*}, \ldots, x_{n}^{*}\right] .
$$

Consider the Lagrangian function $l(x, \pi)=f(x)-\pi g(x)$. Let $f^{l}$ and $f^{u}, g^{l}$ and $g^{u}$ be the estimated minimum and maximum values of $f$ and $g$, respectively, over $\left[x^{l}, x^{u}\right]$. Also, let $\pi^{*}$ denote the set of marginals obtained at the relaxation of the current node. Then, the weighted violation of $x_{j}$ is computed as

$$
\beta_{x_{j}}\left\{f^{u}-f^{l}+\sum_{i=1}^{m} \pi_{i}^{*}\left(\max \left\{0, g^{l}\right\}-g^{u}\right)+\alpha_{x_{j}} v_{x_{j}}\right\}
$$

where $\alpha_{x_{j}}$ and $\beta_{x_{j}}$ are appropriately chosen constants (branching priorities). In the case of linear relaxations of the form $\min \{c x \mid A x \leq b\}$, the above may be approximated as:

$$
\beta_{x_{j}}\left\{\left|c_{j}\right| v_{x_{j}}+\sum_{i=1}^{m}\left|\pi_{i}^{*} A_{i j}\right| v_{x_{j}}+\alpha_{x_{j}} v_{x_{j}}\right\} .
$$

The branching variable is chosen to be the one with the largest weighted violation. 


\subsection{Branching Point Selection}

Following the choice of the branching variable, the branching point is typically chosen by an application of the bisection rule, $\omega$-rule, or their modified versions $[31,35,38]$. In Section 3.2, we showed that, for any univariate convex function, the gap between the over- and under-estimators reduces as $O\left(1 / n^{2}\right)$, provided that the branching point is chosen via the interval bisection rule, slope bisection rule, projective rule, maximum error rule, chord rule, or the angular bisection rule. The branch-and-bound algorithm operates in a manner similar to the sandwich algorithm as it identifies and subsequently refines regions where the function is desired to be approximated more closely. However, in branch-andbound, those regions are chosen where the lower bounding function attains the minimum value. In contrast, in the sandwich algorithm, the error measure between the function and its approximating functions is used for the selection of the interval for further refinement. Still, in the course of our branch-andbound, the approximation error of a function reduces by $O\left(1 / n^{2}\right)$. Here, $n$ is the number of times partitioning was done along the variable axis corresponding to the independent variable in all ancestors of the current node.

As an alternative strategy, a bilinear program for branching point selection is given in [38]. For a given branching variable, this formulation identifies a branching point that locally maximizes the improvement in the lower bound for the current node.

\section{Tree Navigation and Expansion}

At every iteration, branch-and-bound chooses a node from the list of open nodes for processing. The choice of this node governs the structure of the branch-andbound tree explored before convergence is attained. This choice also determines to a large extent the memory requirements of the algorithm.

The node selection rule we employ is a composite rule based on a priority ordering of the nodes. Priority orderings based on lower bound, violation, and order of creation are dynamically maintained during the search process. The violation of a node is defined as the summation of the errors contributed by each variable of a branch-and-bound node (see Section 5.1). The default node selection rule switches between the node with the minimum violation and the one with least lower bound. When memory limitations become stringent, we temporarily switch to the LIFO rule.

While solving large problems using branch-and-bound methods, it is not uncommon to generate a large number of nodes in the search tree. Let us say that the number of open nodes in the branch-and-bound tree at a given point in time is $L$. The naive method of traversing the list of nodes to select the node satisfying the priority rule takes $\Theta(L)$ time. Unlike most other procedures conducted at every node of the branch-and-bound tree, the node selection process consumes time that is super-polynomial in the size of the input. It therefore pays to use more sophisticated data structures for maintaining priority queues. 
In particular, we maintain the priority orderings using the heap data structure for which insertion and deletion of a node may be done in $O(\log L)$ time and retrieval of the node satisfying the priority rule takes only $O(1)$ time. In particular, for combinatorial optimization problems, the node selection rule is guaranteed to spend time linear in the size of the input.

Once the current node has been solved and a branching decision has been taken, the branching decision is stored and the node is partitioned only when it is chosen subsequently by the node selection rule. This approach is motivated by two reasons. First, it results in half the memory requirements as compared to the case in which the node is partitioned immediately after branching. Secondly, postponement offers the possibility of partial optimization of a node's relaxation. For example, consider a relaxation that is solved using an outer-approximation method based on Lagrangian duality, Benders decomposition, or a dual ascent procedure such as bundle methods. In such cases, it may not be beneficial to solve the relaxation to optimality if, after a certain number of iterations, a lower bound is proven for this node which significantly reduces the priority of this node. When this node is picked again for further exploration, the relaxation may be solved to optimality. A similar scheme is followed if range reduction leads to significant tightening of the variable bounds.

\section{Implementation and Computational Results}

The Branch-and-Reduce Optimization Navigator (BARON) [32] is an implementation of our branch-and-bound algorithm for global optimization problems. BARON is modular with problem-specific enhancements (modules) that are isolated from the core implementation of the branch-and-bound algorithm.

BARON's core consists of nine major components: preprocessor, navigator, data organizer, I/O handler, range reduction utilities, sparse matrix utilities, solver links, automatic gradient and function evaluator, and debugging facilities:

- The navigator - the principal component of BARON - coordinates the transitions between the preidentified computation states of a branch-andbound algorithm, including node preprocessing, lower bounding, range reduction, upper bounding, node postprocessing, branching, and node selection. The execution of the navigator can be fine-tuned using a wide variety of options available to the user. For example, the user may choose between various node selection schemes like LIFO, best bound, node violation, and other composite rules based on these priority orders. The navigator calls the module-specific range reduction, lower bounding, upper bounding, feasibility checker, and objective function evaluator during the appropriate phases of the algorithm.

- The data organizer is tightly integrated with the navigator and maintains the branch-and-bound tree structure, priority queues, and bases information to hot-start the solver at every node of the search tree. These data structures are maintained in a linear work array. Therefore, the data 
organizer provides its own memory management facilities (allocator and garbage collector) which are suited to the branch-and-bound algorithm. Data compression techniques are used for storing bounded integer arrays like the bounds of integer variables and the LP basis. Each module is allowed to store its problem-specific information in the work-array before the computation starts at every node of the branch-and-bound tree.

- The I/O handler provides the input facilities for reading in the problem and the options that affect the execution of the algorithm. A contextfree grammar has been developed for the input of factorable nonlinear programming problems where the constraint and objective functions are recursive sums and products of univariate functions of monomials, powers, and logarithms. In addition, each module supports its own input format through its input routine. Output routines provide the timing details and a summary of the execution in addition to the solutions encountered during the search process. The output routines may also be instructed to dump detailed information from every step of the branch-and-bound algorithm for debugging purposes.

- The range reduction facilities include feasibility-based tightening, marginalsbased range reduction, probing, duality-based tightening schemes, and interval arithmetic utilities for logarithmic, monomial, power, and bilinear functions of variables.

- BARON has interfaces to various solvers like OSL, CPLEX, MINOS, SNOPT, and SDPA. BARON also provides automatic differentiation routines to interface to the nonlinear solvers. The interfaces save the requisite hot-starting information from the solver and use it at subsequent nodes in the tree. Depending on the degree of change in the problem fed to the solver, an appropriate update scheme is automatically chosen by each interface. The interfaces also provide the I/O handler with the solver-specific information that may be desired by the user for debugging purposes.

In addition to the core component, the BARON library includes solvers for mixed-integer linear programming, separable concave quadratic programming, indefinite quadratic programming, separable concave programming, linear multiplicative programming, general linear multiplicative programming, univariate polynomial programming, $0-1$ hyperbolic programming, integer fractional programming, fixed-charge programming, power economies of scale, mixed-integer semidefinite programming, and factorable nonlinear programming. In particular, what we refer to as the factorable NLP module of BARON is the most general of the modules and addresses the solution of factorable NLPs with or without additional integrality requirements. Without any user intervention, BARON constructs linear/nonlinear relaxations and solves relaxations while at the same time performing local optimization using widely available solvers.

Extensive computational experience with the proposed algorithm on over 500 problems is reported in [38]. Below, we present computational results on three 


\begin{tabular}{|ccc|ccc|crr|rrr|}
\hline & & & \multicolumn{3}{|c|}{ BARON SCQP } & \multicolumn{3}{c|}{ BARON SCQP } & \multicolumn{3}{c|}{ BARON NLP } \\
norobing & \multicolumn{3}{c|}{ no probing } \\
Problem & $m$ & $n$ & $T_{\text {tot }}$ & $N_{\text {tot }}$ & $N_{\text {mem }}$ & $T_{\text {tot }}$ & $N_{\text {tot }}$ & $N_{\text {mem }}$ & $T_{\text {tot }}$ & $N_{\text {tot }}$ & $N_{\text {mem }}$ \\
\hline FP7a & 10 & 20 & 0.17 & 77 & 5 & 0.28 & 71 & 4 & 1.38 & 51 & 6 \\
FP7b & 10 & 20 & 0.19 & 93 & 7 & 0.25 & 59 & 4 & 1.38 & 51 & 6 \\
FP7c & 10 & 20 & 0.19 & 81 & 7 & 0.28 & 71 & 4 & 1.29 & 45 & 6 \\
FP7d & 10 & 20 & 0.19 & 81 & 4 & 0.25 & 65 & 4 & 1.26 & 49 & 6 \\
FP7e & 10 & 20 & 0.38 & 197 & 16 & 0.56 & 141 & 12 & 8.87 & 339 & 30 \\
RV1 & 5 & 10 & 0.04 & 35 & 3 & 0.04 & 11 & 3 & 0.29 & 29 & 3 \\
RV2 & 10 & 20 & 0.16 & 103 & 7 & 0.20 & 37 & 5 & 1.37 & 65 & 8 \\
RV3 & 20 & 20 & 0.40 & 233 & 15 & 0.28 & 73 & 10 & 3.22 & 141 & 11 \\
RV7 & 20 & 30 & 0.32 & 155 & 6 & 0.23 & 41 & 4 & 3.63 & 109 & 7 \\
RV8 & 20 & 40 & 0.47 & 187 & 13 & 0.45 & 58 & 8 & 3.1 & 81 & 8 \\
RV9 & 20 & 50 & 1.25 & 526 & 38 & 1.19 & 184 & 31 & 15.9 & 331 & 34 \\
M1 & 11 & 20 & 0.36 & 189 & 3 & 0.61 & 151 & 3 & 2.11 & 99 & 7 \\
M2 & 21 & 30 & 0.93 & 291 & 3 & 1.67 & 233 & 2 & 5.57 & 159 & 2 \\
\hline
\end{tabular}

Table 1: Computational results for small SCQPs

representative classes of problems: a purely continuous nonlinear class, a purely integer nonlinear class, and miscellaneous mixed-integer nonlinear programs. The machine on which we perform all of our computations is a $332 \mathrm{MHz}$ RS/6000 Model 43P running AIX 4.3 with 128MB RAM and a LINPACK score of 59.9. For all problems solved, we report the total CPU seconds taken to solve the problem $\left(T_{\text {tot }}\right)$, the total number of nodes in the branch-and-bound tree $\left(N_{\text {tot }}\right)$, and the maximum number of nodes that had to be stored in memory during the search $\left(N_{\text {mem }}\right)$. Unless otherwise noted, computations are carried out with an absolute termination tolerance (difference between upper and lower bounds) of $\epsilon_{a}=10^{-6}$. BARON converged with a global optimal solution within this tolerance for all problems reported in this paper.

\subsection{Separable Concave Quadratic Programs (SCQPs)}

In Table 1, we present computational results for the most difficult of the separable concave quadratic problems in [35]. In this table, $m$ and $n$, respectively, denote the number of constraints and variables of the problems solved. We use the BARON SCQP module with and without probing and the NLP module without probing to solve these problems. As seen in this table, our general purpose factorable NLP module takes approximately the same number of branch-andbound iterations to solve the problems as the specialized SCQP module.

The problems tackled in Table 1 are relatively small. We next provide computational results on larger problems that are generated using the test problem 


\begin{tabular}{|c|c|c|c|c|c|c|c|c|c|c|c|c|c|}
\hline \multirow{2}{*}{\multicolumn{3}{|c|}{$\begin{array}{l}\text { Algorithm } \\
\text { Computer }\end{array}$}} & \multirow{4}{*}{$\begin{array}{c}\text { GOP96 [42] } \\
\text { HP9000/730 } \\
T_{\text {tot }} \\
\text { avg }\end{array}$} & \multirow{4}{*}{$\begin{array}{c}\text { GOP/MILP }[42] \\
\text { HP9000/730 } \\
T_{\text {tot }} \\
\text { avg }\end{array}$} & \multicolumn{9}{|c|}{ BARON SCQP } \\
\hline & & & & & \multicolumn{9}{|c|}{$\mathrm{RS} 6000 / 43 \mathrm{P}$} \\
\hline & & & & & \multicolumn{3}{|c|}{$T_{\text {tot }}$} & \multicolumn{3}{|c|}{$N_{\text {tot }}$} & \multicolumn{3}{|c|}{$N_{\text {mem }}$} \\
\hline$m$ & $n$ & $k$ & & & $\min$ & avg & $\max$ & $\min$ & avg & $\max$ & $\min$ & avg & $\max$ \\
\hline 50 & 50 & 50 & 0.12 & 0.12 & 0.10 & 0.10 & 0.10 & 1 & 2 & 3 & 1 & 1 & 2 \\
\hline 50 & 50 & 100 & 0.15 & 0.14 & 0.10 & 0.22 & 0.30 & 1 & 13 & 24 & 1 & 5 & 9 \\
\hline 50 & 50 & 200 & 6.05 & 1.57 & 0.30 & 0.70 & 1.60 & 9 & 51 & 170 & 5 & 17 & 45 \\
\hline 50 & 50 & 500 & - & 14.13 & 1.00 & 1.86 & 2.80 & 21 & 68 & 108 & 9 & 16 & 30 \\
\hline 50 & 100 & 100 & 0.22 & 1.37 & 0.30 & 0.72 & 1.10 & 20 & 62 & 127 & 6 & 23 & 48 \\
\hline 50 & 100 & 200 & 0.36 & 11.98 & 0.50 & 1.48 & 3.30 & 17 & 98 & 246 & 4 & 20 & 39 \\
\hline 100 & 100 & 100 & 0.31 & 0.31 & 0.40 & 0.46 & 0.50 & 3 & 7 & 14 & 2 & 4 & 8 \\
\hline 100 & 100 & 200 & 0.38 & 0.36 & 0.70 & 0.78 & 0.90 & 14 & 26 & 37 & 5 & 8 & 11 \\
\hline 100 & 100 & 500 & - & 80.03 & 1.61 & 4.40 & 15.2 & 43.7 & 740 & 2314 & 44 & 161 & 425 \\
\hline 100 & 150 & 400 & - & 180.2 & 1.20 & 18.8 & 82.6 & 8 & 927 & 4311 & 3 & 163 & 721 \\
\hline
\end{tabular}

Table 2: Comparative computational results for large SCQPs

generator of [28]. The problems generated are of the form:

$$
\begin{array}{ll}
\min & \frac{1}{2} \theta_{1} \sum_{j=1}^{n} \lambda_{j}\left(x_{j}-\bar{\omega}_{j}\right)^{2}+\theta_{2} \sum_{j=1}^{k} d_{j} y_{j} \\
\text { s.t. } & A_{1} x+A_{2} y \leq b \\
& x \geq 0, y \geq 0
\end{array}
$$

where $x \in \mathbb{R}^{n}, \lambda \in \mathbb{R}^{n}, \bar{\omega} \in \mathbb{R}^{n}, y \in \mathbb{R}^{k}, d \in \mathbb{R}^{k}, b \in \mathbb{R}^{m}, A_{1} \in \mathbb{R}^{m \times n}$, $A_{2} \in R^{m \times k}, \theta_{1}=-0.001$, and $\theta_{2}=0.1$. The results of Table 2 were obtained by solving 5 randomly generated instances for every problem size. The algorithms compared in Table 2 used a relative termination tolerance of $\epsilon_{r}=0.1$. Our algorithm is up to an order of magnitude faster than that of [42] for large problems.

\subsection{Cardinality Constrained Hyperbolic Programming}

Cardinality constrained hyperbolic programs are of the following form:

$$
\begin{aligned}
(\mathrm{CCH}) \quad \max & \sum_{i=1}^{m} \frac{a_{i 0}+\sum_{j=1}^{n} a_{i j} x_{j}}{b_{i 0}+\sum_{j=1}^{n} b_{i j} x_{j}} \\
\text { s.t. } \quad & \sum_{j=1}^{n} x_{j}=p \\
& \sum_{j=1}^{n} b_{i 0}+b_{i j} x_{j}>0 \quad i=1, \ldots, m \\
& x_{j} \in\{0,1\} \quad j=1, \ldots, n,
\end{aligned}
$$


where $a \in \mathbb{R}^{n+1}$ and $b \in \mathbb{R}^{n+1}$ for $i=1, \ldots, m, p \in \mathbb{Z}, 0 \leq p \leq n$.

As we detail in [39], we can convert the pure nonlinear integer program $\mathrm{CCH}$ to an MILP using the reformulation strategy shown below where $v_{i j}=g_{i} x_{j}$ is enforced by using the bilinear envelopes of [25]:

$$
\begin{aligned}
& \text { (R7) } \max \sum_{i=1}^{m} g_{i} \\
& \text { s.t. } \sum_{j=1}^{n} x_{j}=p \\
& \sum_{j=1}^{n} v_{i j}=g_{i} p \quad i=1, \ldots, m \\
& b_{i 0} g_{i}+\sum_{j=1}^{n} b_{i j} v_{i j}=a_{i 0}+\sum_{j=1}^{n} a_{i j} x_{j} \quad i=1, \ldots, m \\
& v_{i j} \leq g_{i}^{u} x_{j} \quad i=1, \ldots, m ; j=1, \ldots, n ; b_{i j}<0 \\
& v_{i j} \leq g_{i}+g_{i}^{l} x_{j}-g_{i}^{l} \quad i=1, \ldots, m ; j=1, \ldots, n ; b_{i j}<0 \\
& v_{i j} \geq g_{i}+g_{i}^{u} x_{j}-g_{i}^{u} \quad i=1, \ldots, m ; j=1, \ldots, n ; b_{i j}>0 \\
& v_{i j} \geq g_{i}^{l} x_{j} \quad i=1, \ldots, m ; j=1, \ldots, n ; b_{i j}>0 \\
& x \in\{0,1\}^{n}
\end{aligned}
$$

We derive tight bounds on $g_{i}$ using an algorithm devised by Saipe in [33]. Then, we use BARON to carry out the above reformulation at every node of the tree to solve $\mathrm{CCH}$ and compare the performance of our algorithm against that of CPLEX on the MILP. As Table 3 shows, range contraction and reformulation at every node results in a much superior approach.

\begin{tabular}{|rrr|rrr|rrr|}
\hline \multicolumn{3}{|c|}{ Problem } & \multicolumn{3}{c|}{ CPLEX 6.0 } & \multicolumn{3}{c|}{ BARON HYP } \\
\hline$m$ & $n$ & $p$ & $T_{\text {tot }}$ & $N_{\text {tot }}$ & $N_{\text {mem }}$ & $T_{\text {tot }}$ & $N_{\text {tot }}$ & $N_{\text {mem }}$ \\
\hline 5 & 20 & 12 & 1.07 & 40 & 39 & 1.9 & 11 & 4 \\
10 & 20 & 8 & 105 & 1521 & 1518 & 70.5 & 234 & 54 \\
10 & 20 & 12 & 7.4 & 96 & 95 & 11.2 & 31 & 7 \\
20 & 30 & 14 & 22763 & 47991 & 47076 & 1811 & 1135 & 216 \\
20 & 20 & 12 & 207 & 666 & 632 & 83 & 85 & 18 \\
20 & 30 & 16 & 17047 & 38832 & 36904 & 1912 & 1353 & 256 \\
5 & 50 & 25 & 19372 & 506225 & 184451 & 2534 & 6959 & 1230 \\
5 & 50 & 27 & - & - & - & 1803 & 5079 & 893 \\
\hline \multicolumn{8}{|c}{ " in this table indicates a problem for which } \\
\hline
\end{tabular}

Table 3: Computational results for $\mathrm{CCH}$

\subsection{Mixed-Integer Nonlinear Programs from minlplib}

In this section, we address the solution of mixed-integer nonlinear programs. In particular, we focus our attention on a collection of MINLPs from minlplib [8], which provides complete model descriptions and references to original sources for all problems solved in this section. 
The computational results are presented in Table 4 where, for each problem, we report the globally optimal objective function value, the number of problem constraints $(m)$, the total number of problem variables $(n)$, and the number of discrete variables $\left(n_{\mathrm{d}}\right)$, in addition to $\mathrm{CPU}$ and node information. Problems nvs01 through nvs24 originate from [16] who used local solutions to nonconvex nonlinear programs at each node and conducted branch-and-bound on the integer variables. Even though the problem sizes are small, the solutions we obtained for problems nvs02, nvs14, nvs20, and nvs21 correspond to significantly better objective function values than those reported in the original source of these problems [16], demonstrating the benefits of global optimization.

Amongst the remaining problems of this table, one finds a nonlinear fixedcharge problem (st_e27), a reliability problem (st_e29), a mechanical fixture design problem (st_e31), a heat exchanger network synthesis problem (st_e35), a pressure design problem (st_e38), a truss design problem (st_e40), a problem from the design of just-in-time manufacturing systems (jit), and a particulary challenging molecular design problem (primary).

\section{Conclusions}

The subject matter of this paper has been the development and implementation of a global optimization algorithm for mixed-integer nonlinear programming. Our algorithmic developments included a new outer-approximation scheme that provides an entirely LP-based relaxation of MINLPs, a new theoretical framework for domain reduction, and new branching strategies. Computational results with our branch-and-bound implementation in BARON demonstrated the flexibility that such a computational model offers in solving MINLPs from a variety of disciplines through an entirely automated procedure.

\section{Acknowledgments}

The authors are grateful to two anonymous referees whose constructive comments helped improve the presentation of this manuscript significantly.

\section{References}

[1] S. Ahmed, M. Tawarmalani, and N. V. Sahinidis. A finite branch and bound algorithm for two-stage stochastic integer programs. Mathematical Programming. Submitted, 2000.

[2] F. A. Al-Khayyal and J. E. Falk. Jointly constrained biconvex programming. Mathematics of Operations Research, 8:273-286, 1983.

[3] F. A. Al-Khayyal and H. D. Sherali. On finitely terminating branch-andbound algorithms for some global optimization problems. SIAM Journal on Optimization, 10:1049-1057, 2000. 


\begin{tabular}{|c|c|c|c|c|c|c|c|c|}
\hline Problem & & Obj. & $m$ & $n$ & $n_{\mathrm{d}}$ & $T_{\text {tot }}$ & $N_{\text {tot }}$ & $N_{\text {mem }}$ \\
\hline nvs01 & & 12.47 & 3 & 3 & 2 & 0.05 & 29 & 5 \\
\hline nvs02 & * & 5.96 & 3 & 8 & 5 & 0.04 & 13 & 6 \\
\hline nvs03 & & 16.00 & 3 & 2 & 2 & 0.01 & 3 & 2 \\
\hline nvs04 & & 0.72 & 0 & 2 & 2 & 0.02 & 1 & 1 \\
\hline nvs05 & & 5.47 & 9 & 8 & 2 & 1.91 & 241 & 52 \\
\hline nvs06 & & 1.77 & 0 & 2 & 2 & 0.03 & 11 & 5 \\
\hline nvs07 & & 4.00 & 2 & 3 & 3 & 0.02 & 3 & 2 \\
\hline nvs08 & & 23.45 & 3 & 3 & 2 & 0.39 & 21 & 5 \\
\hline nvs09 & & -43.13 & 0 & 10 & 10 & 0.16 & 39 & 7 \\
\hline nvs10 & & -310.80 & 2 & 2 & 2 & 0.04 & 12 & 4 \\
\hline nvs11 & & -431.00 & 3 & 3 & 3 & 0.07 & 34 & 8 \\
\hline nvs12 & & -481.20 & 4 & 4 & 4 & 0.11 & 70 & 13 \\
\hline nvs13 & & -585.20 & 5 & 5 & 5 & 0.40 & 208 & 30 \\
\hline nvs14 & $*$ & -40358.20 & 3 & 8 & 5 & 0.03 & 8 & 5 \\
\hline nvs15 & & 1.00 & 1 & 3 & 3 & 0.02 & 11 & 3 \\
\hline nvs16 & & 0.70 & 0 & 2 & 2 & 0.03 & 23 & 12 \\
\hline nvs17 & & -1100.40 & 7 & 7 & 7 & 12.00 & 3497 & 388 \\
\hline nvs18 & & -778.40 & 6 & 6 & 6 & 2.75 & 1037 & 122 \\
\hline nvs19 & & -1098.40 & 8 & 8 & 8 & 43.45 & 9440 & 1022 \\
\hline nvs20 & $*$ & 230.92 & 2 & 3 & 2 & 1.64 & 156 & 19 \\
\hline nvs21 & * & -5.68 & 2 & 3 & 2 & 0.05 & 31 & 3 \\
\hline nvs22 & & 6.06 & 9 & 8 & 4 & 0.08 & 14 & 6 \\
\hline nvs23 & & -1125.20 & 9 & 9 & 9 & 168.23 & 25926 & 3296 \\
\hline nvs24 & & -1033.20 & 10 & 10 & 10 & 1095.87 & 130487 & 15967 \\
\hline st_e27 & & 2.00 & 2 & 1 & 1 & 0.02 & 3 & 2 \\
\hline st_e29 & & -0.94 & 6 & 2 & 2 & 0.18 & 47 & 11 \\
\hline st_e31 & & -2.00 & 135 & 112 & 24 & 3.75 & 351 & 56 \\
\hline st_e32 & & -1.43 & 18 & 35 & 19 & 13.7 & 906 & 146 \\
\hline st_e35 & & 64868.10 & 39 & 32 & 7 & 16.4 & 465 & 57 \\
\hline st_e36 & & -246.00 & 2 & 2 & 1 & 2.59 & 768 & 72 \\
\hline st_e38 & & 7197.73 & 3 & 4 & 2 & 0.38 & 5 & 2 \\
\hline st_e40 & & 30.41 & 7 & 4 & 3 & 0.15 & 24 & 6 \\
\hline jit & & 173,983 & 33 & 26 & 4 & 0.67 & 63 & 10 \\
\hline primary & & -1.2880 & 164 & 82 & 58 & 375 & 15930 & 1054 \\
\hline
\end{tabular}

Table 4: Selected MINLP problems from [8] 
[4] M. S. Bazaraa, H. D. Sherali, and C. M. Shetty. Nonlinear Programming, Theory and Algorithms. Wiley Interscience, Series in Discrete Mathematics and Optimization, 2nd edition, 1993.

[5] D. Bienstock. Computational study of a family of mixed-integer quadratic programming problems. Mathematical Programming, 74:121-140, 1996.

[6] B. Borchers and J. E. Mitchell. An improved branch and bound for mixed integer nonlinear programs. Comput. Oper. Res., 21:359-367, 1994.

[7] B. Borchers and J. E. Mitchell. A computational comparison of branch and bound and outer approximation algorithms for 0-1 mixed integer nonlinear programs. Comput. Oper. Res., 24:699-701, 1997.

[8] M. R. Bussieck and A. Meeraus. MINLPLib-A Collection of Test Models for Mixed-Integer Nonlinear Programming. GAMS Development Corporation, Washington D.C., 2001.

[9] R. J. Dakin. A tree search algorithm for mixed integer programming problems. Computer Journal, 8:250-255, 1965.

[10] M. A. Duran and I. E. Grossmann. An outer-approximation algorithm for a class of mixed-integer nonlinear programs. Mathematical Programming, 36:307-339, 1986.

[11] J. E. Falk and R. M. Soland. An algorithm for separable nonconvex programming problems. Management Science, 15:550-569, 1969.

[12] C. A. Floudas. Deterministic Global Optimization: Theory, Algorithms and Applications. Kluwer Academic Publishers, Dordrecht, 1999.

[13] B. Fruhwirth, R. E. Burkard, and G. Rote. Approximation of convex curves with applications to the bicriteria minimum cost flow problem. European Journal of Operational Research, 42:326-338, 1989.

[14] P. M. Gruber. Aspects of approximation of convex bodies. In P. M. Gruber and J. M. Wills (eds.), Handbook of Convex Geometry, North-Holland, 1993.

[15] P. M. Gruber and P. Kenderov. Approximation of convex bodies by polytopes. Rendiconti Circ. Mat. Palermo, Serie II, 31:195-225, 1982.

[16] O. K. Gupta and A. Ravindran. Branch and bound experiments in convex nonlinear integer programming. Management Science, 31:1533-1546, 1985.

[17] A. S. E. Hamed and G. P. McCormick. Calculation of bounds on variables satisfying nonlinear inequality constraints. Journal of Global Optimization, 3:25-47, 1993.

[18] P. Hansen, B. Jaumard, and S.-H. Lu. An analytic approach to global optimization. Mathematical Programming, 52:227-254, 1991. 
[19] R. Horst and H. Tuy. Global Optimization: Deterministic Approaches. Springer Verlag, Berlin, Third edition, 1996.

[20] B. W. Lamar. An improved branch and bound algorithm for minimum concave cost network flow problems. Journal of Global Optimization, 3:261287, 1993.

[21] A. H. Land and A. G. Doig. An automatic method for solving discrete programming problems. Econometrica, 28:497-520, 1960.

[22] R. Lazimy. Mixed-integer quadratic programming. Mathematical Programming, 22:332-349, 1982.

[23] R. Lazimy. Improved algorithm for mixed-integer quadratic programs and a computational study. Mathematical Programming, 32:100-113, 1985.

[24] R. D. McBride and J. S. Yormark. An implicit enumeration algorithm for quadratic integer programming. Management Science, 26:282-296, 1980.

[25] G. P. McCormick. Computability of global solutions to factorable nonconvex programs: Part I-Convex underestimating problems. Mathematical Programming, 10:147-175, 1976.

[26] G. P. McCormick. Nonlinear Programming: Theory, Algorithms and Applications. John Wiley \& Sons, 1983.

[27] G. L. Nemhauser and L. A. Wolsey. Integer and Combinatorial Optimization. Wiley Interscience, Series in Discrete Mathematics and Optimization, 1988.

[28] A. T. Phillips and J. B. Rosen. A parallel algorithm for constrained concave quadratic global minimization. Mathematical Programming, 42:421-448, 1988.

[29] G. Rote. The convergence rate of the sandwich algorithm for approximating convex functions. Computing, 48:337-361, 1992.

[30] H. S. Ryoo and N. V. Sahinidis. Global optimization of nonconvex NLPs and MINLPs with applications in process design. Computers \&5 Chemical Engineering, 19:551-566, 1995.

[31] H. S. Ryoo and N. V. Sahinidis. A branch-and-reduce approach to global optimization. Journal of Global Optimization, 8:107-139, 1996.

[32] N. V. Sahinidis. BARON: A general purpose global optimization software package. Journal of Global Optimization, 8:201-205, 1996.

[33] A. L. Saipe. Solving a $(0,1)$ hyperbolic program by branch and bound. Naval Research Logistics Quarterly, 22:497-515, 1975. 
[34] M. W. P. Savelsbergh. Preprocessing and probing for mixed integer programming problems. ORSA Journal on Computing, 6:445-454, 1994.

[35] J. P. Shectman and N. V. Sahinidis. A finite algorithm for global minimization of separable concave programs. Journal of Global Optimization, 12:1-36, 1998.

[36] H. D. Sherali and H. Wang. Global optimization of nonconvex factorable programming problems. Mathematical Programming, 89:459-478, 2001.

[37] E. M. B. Smith and C. C. Pantelides. Global optimisation of general process models. In I. E. Grossmann (ed.), Global Optimization in Engineering Design, Kluwer Academic Publishers, Boston, MA, pages 355-386, 1996.

[38] M. Tawarmalani. Mixed Integer Nonlinear Programs: Theory, Algorithms and Applications. PhD thesis, Department of Mechanical \& Industrial Engineering, University of Illinois, Urbana-Champaign, IL, August 2001.

[39] M. Tawarmalani, S. Ahmed, and N. V. Sahinidis. Global optimization of 0-1 hyperbolic programs. Journal of Global Optimization, 24:385-417, 2002 .

[40] M. Tawarmalani and N. V. Sahinidis. Convex extensions and convex envelopes of l.s.c. functions. Mathematical Programming, 93:247-263, 2002.

[41] L. S. Thakur. Domain contraction in nonlinear programming: Minimizing a quadratic concave function over a polyhedron. Mathematics of Operations Research, 16:390-407, 1990.

[42] V. Visweswaran and C. A. Floudas. Computational results for an efficient implementation of the GOP algorithm and its variants. In I. E. Grossmann (ed.), Global Optimization in Engineering Design, Kluwer Academic Publishers, Boston, MA, pages 111-153, 1996.

[43] J. M. Zamora and I. E. Grossmann. A branch and contract algorithm for problems with concave univariate, bilinear and linear fractional terms. Journal of Global Optimization, 14:217-249, 1999. 